\title{
Simultaneous Characterization of Sympathetic and Cardiac Arms of the Baroreflex through Sequence Techniques during Incremental Head-Up Tilt
}

\author{
Andrea Marchi ${ }^{1,2}$, Vlasta Bari ${ }^{3}$, Beatrice De Maria ${ }^{1,4}$, Murray Esler $^{5}$, Elisabeth Lambert ${ }^{5}$, \\ Mathias Baumert ${ }^{6}$ and Alberto Porta ${ }^{3,7 *}$ \\ ${ }^{1}$ Department of Electronics Information and Bioengineering, Politecnico di Milano, Milan, Italy, ${ }^{2}$ Department of Emergency \\ and Intensive Care, San Gerardo Hospital, Monza, Italy, ${ }^{3}$ Department of Biomedical Sciences for Health, University of Milan, \\ Milan, Italy, ${ }^{4}$ IRCCS Fondazione Salvatore Maugeri, Milan, Italy, ${ }^{5}$ Human Neurotransmitter Laboratory, Baker IDI Heart and \\ Diabetes Institute, Melbourne, VIC, Australia, ${ }^{6}$ School of Electrical and Electronic Engineering, University of Adelaide, \\ Adelaide, SA, Australia, ${ }^{7}$ Department of Cardiothoracic, Vascular Anesthesia and Intensive Care, IRCCS Policlinico San \\ Donato, Milan, Italy
}

OPEN ACCESS

Edited by:

Zbigniew R. Struzik,

University of Tokyo, Japan

Reviewed by:

Jens Tank

Hannover Medical School, Germany

André Diedrich,

Vanderbilt University, USA

Satoshi Iwase,

Aichi Medical University, Japan

*Correspondence:

Alberto Porta

alberto.porta@unimi.it

Specialty section:

This article was submitted to Computational Physiology and

Medicine,

a section of the journal

Frontiers in Physiology

Received: 28 June 2016 Accepted: 14 September 2016 Published: 29 September 2016

Citation:

Marchi A, Bari V, De Maria B, Esler M, Lambert E, Baumert M and Porta $A$ (2016) Simultaneous Characterization

of Sympathetic and Cardiac Arms of the Baroreflex through Sequence

Techniques during Incremental

Head-Up Tilt. Front. Physiol. 7:438

doi: 10.3389/fphys.2016.00438
We propose a sympathetic baroreflex (sBR) sequence method for characterizing sBR from spontaneous beat-to-beat fluctuations of muscle sympathetic nerve activity (MSNA) and diastolic arterial pressure (DAP). The method exploits a previously defined MSNA variability quantifying the fluctuations of MSNA burst rate. The method is based on the detection of MSNA and DAP sequences characterized by the contemporaneous DAP increase and MSNA decrease or vice versa. The percentage of sBR sequences (SEQ\% $\%_{s B R}$ ) was taken as an indication of the degree of sBR solicitation and the average slope of the regression lines in the (DAP, MSNA) plane was taken as sBR sensitivity (sBRS $S_{S E Q}$ ) and expressed in bursts $\mathrm{s}^{-1} \cdot \mathrm{mmHg}^{-1}$. $\mathrm{sBRS} \mathrm{SEQ}_{\mathrm{SE}}$ was compared to a more traditional estimate based on the baroreflex threshold analysis (sBRS $\mathrm{BTA}_{\mathrm{B} A}$ ). An incremental head-up tilt protocol, carried out in 12 young healthy subjects (age: 20-36 yr, median $=22.5 \mathrm{yr}, 9$ females) sequentially tilted at $0,20,30,40,60^{\circ}$ table inclinations, was utilized to set the sBR sequence method parameters. Traditional sequence analysis was exploited to estimate cardiac baroreflex (CBR) sensitivity $\left(\mathrm{CBRS}_{\mathrm{SEQ}}\right)$ and percentage of $\mathrm{cBR}$ sequences $\left(\mathrm{SEQ} \%_{\mathrm{CBR}}\right)$. The head-up tilt induced the progressive increase of SEQ\% ${ }_{S B R}$ and SEQ\% ${ }_{C B R}$ and gradual decrease of both $S B R S_{S E Q}$ and ${ }_{C B R S}$ SEQ, thus suggesting the gradual rise of the SBR and CBR solicitations and the progressive reduction of their effectiveness with the stimulus. SBRS $S_{S E Q}$ was significantly associated with sBRS $S_{B T A}$. sBRS $S_{S E Q}$ and $c B R S_{S E Q}$ were significantly correlated as well as $S E Q \%_{S B R}$ and $S E Q \%_{c B R}$, even though the correlation was not strong, thus suggesting a certain degree of independence between the baroreflex arms. The proposed sBR sequence approach provides a dynamical characterization of the sBR alternative to more traditional static pharmacological and nonpharmacological methods and fully homogenous with the cBR sequence technique.

Keywords: heart rate variability, arterial pressure variability, muscle sympathetic nerve activity, autonomic nervous system, cardiovascular control 


\section{INTRODUCTION}

Baroreflex plays a central role in arterial pressure (AP) homeostasis (Robertson et al., 2012) by triggering modifications of many physiological variables including e.g., heart period (HP), sympathetic activity and peripheral resistances in the attempt to buffer AP variations. The arm of the baroreflex acting upon HP, usually referred to as cardiac baroreflex (cBR), is one of the most actively studied (Hunt et al., 2001). The quantitative estimation of the $\mathrm{CBR}$ sensitivity (cBRS) was based on the observation that HP lengthens (or shortens) in response to the AP rise (or fall) induced by a quick, intravenous, administration of a vasoactive drug (Smyth et al., 1969; Pickering et al., 1972). cBRS is measured as the slope of the linear regression of HP on systolic AP (SAP) following the pharmacological challenge. Parallel increases or decreases in both HP and SAP, usually referred to as $\mathrm{cBR}$ sequences, were found even in absence of pharmacologically induced SAP changes when spontaneous HP and SAP variations were contemporaneously observed on a beat-to-beat basis (Bertinieri et al., 1985). This observation allowed the estimation of the cBRS in more physiological conditions from spontaneous variations of HP and SAP as the average slope of the regression lines computed over the $\mathrm{cBR}$ sequences.

CBR is just one arm of the baroreflex. Indeed, since modifications of AP lead to changes of the sympathetic activity that restore AP, a sympathetic arm of the baroreflex has been presumed (Sundlöf and Wallin, 1978). The sympathetic baroreflex (sBR) has been characterized in humans by exploiting microneurographic recordings of integrated muscle sympathetic nerve activity (MSNA). Since the likelihood of a MSNA burst increases while diastolic AP (DAP) decreases, the slope of the linear regression of the percentage of heart beats associated to a MSNA burst at a given DAP value on the DAP value has been proposed to estimate the sBR sensitivity (sBRS) (Sundlöf and Wallin, 1978; Rudas et al., 1999; Kienbaum et al., 2001).

Despite the relevance of evaluating simultaneously sBR and cBR, few studies so far assessed contemporaneously the two different aspects of the same control reflex (Rudas et al., 1999; O'Leary et al., 2003; Dutoit et al., 2010; Taylor et al., 2015). This is the consequence of the difficulties in defining a common framework to assure a homogenous characterization of both $s B R$ and $c B R$. This common framework is difficult to be set when using methods exclusively based on pharmacological interventions (Rudas et al., 1999; Dutoit et al., 2010) or spontaneous physiological variations (O'Leary et al., 2003; Taylor et al., 2015). Indeed, when the modified Oxford method (Ebert

Abbreviations: sBR, sympathetic baroreflex; cBR, cardiac baroreflex; sBRS, sBR sensitivity; $\mathrm{cBRS}, \mathrm{cBR}$ sensitivity; $\mathrm{sBRS} \mathrm{SEQ}_{\mathrm{SE}}$, sBRS computed via the sBR sequence method; $\mathrm{SEQ} \%_{\mathrm{SBR}}$, percentage of $\mathrm{sBR}$ sequences; BTA, baroreflex threshold analysis; $\mathrm{sBRS}_{\mathrm{BTA}}$, sBRS computed via the BTA method; $\mathrm{cBRS} \mathrm{SEQ}_{\mathrm{SEQ}}$, $\mathrm{cBRS}$ computed via the $c B R$ sequence method; $S E Q \%_{c B R}$, percentage of $c B R$ sequences; $E C G$, electrocardiogram; AP, arterial pressure; HP, heart period; SAP, systolic AP; DAP, diastolic AP; MSNA, muscle nerve sympathetic activity; $r$, correlation coefficient; $\mathrm{p}$, type I error probability; $\tau$, lag between physiological variables expressed in beats; T0, T20, T30, T40, T60, head-up tilt with table inclination of $0,20,30,40$, and $60^{\circ}$. and Cowley, 1992) was utilized to compute both sBRS and cBRS (Rudas et al., 1999; Dutoit et al., 2010), their estimates might have different reliability given that at high DAP the likelihood of detecting MSNA burst is null, thus clipping the useful range of AP variations compared to that exploited for the cBRS estimate. On the other hand, when the estimation of both sBRS and cBRS was based on spontaneous variations (O'Leary et al., 2003; Taylor et al., 2015), the comparison is again weak because a dynamical method testing the solicitation of $\mathrm{cBR}$ through the selection of sequences of cBR origin (Bertinieri et al., 1985) was compared to a static method searching for the mere association between binned values of DAP and the occurrence of MSNA burst without assuring that a positive DAP variation produces a negative MSNA burst rate change or vice versa.

The issue of setting a common framework for the contemporaneous assessment of sBRS and cBRS might be easily tackled if baroreflex sequence analysis could be extended to the MSNA and DAP variabilities. Unfortunately, this extension has not been proposed yet due to the unsuitability of the definition of the traditional MSNA variability series. Indeed, since the traditional MSNA variability series is an uncalibrated signal obtained from the integrated MSNA via low-pass filtering preserving exclusively the MSNA oscillations in the range of cardiovascular variability (i.e., from 0 to $0.5 \mathrm{~Hz}$ ), it cannot provide sBRS estimates with suitable units (i.e., bursts $\mathrm{s}^{-1} \cdot \mathrm{mmHg}^{-1}$; Saul et al., 1990; Pagani et al., 1997; Nakata et al., 1998; Taylor et al., 1998; Cooke et al., 1999; Furlan et al., 2000; Kamiya et al., 2005; Ryan et al., 2011) and requires normalization procedures to avoid the dependence of results on factors that are not under control of the investigator (e.g., proximity of the bundle to the recording electrode). Also some classical static methods based on the computation of amplitude or area of the MSNA bursts and its association with binned values of DAP (Sundlöf and Wallin, 1978) require normalization procedures (e.g., the largest MSNA burst amplitude or area is assigned to an arbitrary value and the amplitude or area of all other bursts is expressed in proportion to this value; Kienbaum et al., 2001). This issue can be circumvented by the recent introduction of a calibrated MSNA variability signal (Marchi et al., 2016) focusing on modulations of the burst rate instead of variations of the amplitude of the burst.

The aim of this study is to propose a sBR sequence method for computing sBRS, optimize the parameters of the method by using a graded orthostatic challenge and compare sBRS with cBRS. The study exploits the recently defined calibrated MSNA variability (Marchi et al., 2016) in association with spontaneous DAP variations to compute sBRS, while cBRS is computed over spontaneous HP and SAP variability via the traditional cBR sequence method (Bertinieri et al., 1985). sBRS and cBRS are calculated during the baroreflex unloading induced by an incremental head-up tilt protocol in healthy volunteers (Lambert et al., 2008) imposing a sympathetic activation (Marchi et al., 2016) and a reduction of cBRS (Cooke et al., 1999; Porta et al., 2016) and sBRS (Ichinose et al., 2006; Barbic et al., 2015; Marchi et al., 2015) proportional to the magnitude of the challenge. This study hypothesizes that sBRS decreases in proportion to 
the central hypovolemia induced by incremental head-up tilt and tests a posteriori that the proposed sBR sequence method can monitor this decrease. The sBR sequence method is compared to the baroreflex threshold analysis (BTA), i.e., the traditional static method for the characterization of sBR based on the likelihood of finding a MSNA bust in correspondence of a given DAP value (Sundlöf and Wallin, 1978; Kienbaum et al., 2001; Hart et al., 2010).

\section{METHODS}

\section{Experimental Protocol and Data Acquisition}

The study comprised 12 healthy subjects (age from 20 to $36 \mathrm{yr}$, median $=22.5 \mathrm{yr}$; BMI: from 18.6 to $28.4 \mathrm{~kg} \mathrm{~m}^{-2}$, median $=24.2 \mathrm{~kg}^{-2} ; 9$ females). The study protocol was approved by the Alfred Hospital Ethics Review Committee (n. 144/06) and conformed to the relevant guidelines of the National Health and Medical Research Council of Australia and to the principles of the Declaration of Helsinki for medical research involving humans. All subjects provided written informed consent. The head-up tilt protocol was fully described in Lambert et al. (2008). Briefly, all subjects were tested in the morning, after a light breakfast. Caffeine and alcohol intake was restricted from 7:00 p.m. on the evening before the study. The radial artery was cannulated percutaneously (3F, $5 \mathrm{~cm}$, Cook catheter) to enable invasive AP monitoring. A lead III electrocardiogram (ECG) was recorded via a single lead ECG amplifier (ADInstruments, Castle Hill, NSW, Australia). Respiration movements were monitored via a piezoelectric device (ADInstruments, Castle Hill, NSW, Australia). Multiunit sympathetic nerve firing rates in postganglionic fibers distributed to the skeletal muscle vasculature were recorded by using clinical microneurography via the IOWA Nerve Traffic Analyzer (model 662C-3, Department of Bioengineering, University of Iowa, Iowa; Lambert et al., 2008). After locating the common peroneal nerve, a tungsten microelectrode (FHC, Bowdoinham, Maine) was inserted percutaneously and adjusted until satisfactory spontaneous MSNA was observed in accordance with previously described criteria (Lambert et al., 2008). After instrumentation subjects were allowed to rest for at least $30 \mathrm{~min}$. Then, subjects were sequentially tilted to angles $0,20,30,40$, and $60^{\circ}$ (T0, T20, T30, T40, and T60, respectively) for $10 \mathrm{~min}$ at each angle. The head-up tilt test started from the horizontal position and was incremental with respect to the previous tilt table inclination. ECG, AP, respiratory movements and MSNA were continuously measured at each tilt angle. The raw MSNA signal was band-pass filtered $(700-2000 \mathrm{~Hz})$, amplified, rectified, and integrated (time constant of $0.1 \mathrm{~s}$ ) to obtain integrated MSNA. ECG, AP, respiratory movements and integrated MSNA, were digitized at $1000 \mathrm{~Hz}$ using a PowerLab system (model ML785/8SP, ADInstruments, Castle Hill, NSW, Australia) and stored for off-line analysis. From the respiratory movement signal the respiratory rate was computed. Out of all recordings obtained from the 12 subjects, signals were of insufficient quality or the tilt protocol was not completed, respectively, for one subject during T30 and T40 as well as 5 subjects during T60.

\section{Extraction of the Beat-To-Beat Variability Series}

The R-wave peaks were detected using a traditional method based on a threshold on the first derivative. The jitters in locating the $\mathrm{R}$-wave peak were minimized using parabolic interpolation, thus achieving a time resolution smaller than $1 \mathrm{~ms}$. The temporal distance between two consecutive parabolic apexes was taken as HP. The maximum of AP inside the $i$-th HP was defined as the $i$-th SAP value, where $i$ is the cardiac beat counter. The $i$-th DAP was computed as the minimum of the AP preceding the $i$-th SAP. The occurrences of R-wave peaks and the positions of SAP and DAP fiducial points were carefully checked to avoid erroneous detections or missed beats. The MSNA variability was extracted according to the procedure proposed in Marchi et al. (2016). Briefly, first MSNA bursts were detected from the integrated MSNA signal by using an adaptive thresholding method capable of following on a beat-to-beat basis the baseline wandering and physiological variations of the MSNA burst amplitude (Diedrich et al., 2009). The detection procedure accounted for the latency from the AP sensing to possible MSNA response (Sundlöf and Wallin, 1978; Wallin et al., 1994; Hamner and Taylor, 2001) by searching the MSNA burst in a time window ranging from 0.9 to $1.7 \mathrm{~s}$ starting from the first R-wave peak delimiting the current HP (Kienbaum et al., 2001; Diedrich et al., 2009). After having detected the MSNA burst associated to each R-wave peak, the MSNA bursts were counted over a moving time window of $5 \mathrm{~s}$ that was advanced in steps of $1 \mathrm{~ms}$ along the recording, thus obtaining a step-wise burst-count MSNA signal. Finally, the step-wise burst-count MSNA signal was low-pass filtered with a finite impulse response filter with a cut-off frequency of $0.5 \mathrm{~Hz}$ (8000 coefficients). An example of ECG, AP, integrated MSNA, step-wise burst-count MSNA, and low-pass filtered burst-count MSNA signal recorded from the same subject during T0 and T60 is given in Figure 1. The low-pass filtered burst-count integrated MSNA signal was downsampled once per cardiac beat at the occurrence of the first R-wave peak delimiting the $i$-th HP, thus obtaining a burst-count MSNA beat-to-beat variability series synchronous with HP, SAP, and DAP series. Each value of the burst-count MSNA variability series was divided by the length of the moving time window (i.e., $5 \mathrm{~s}$ ), yielding the MSNA burst rate variability expressed in bursts $\mathrm{s}^{-1}$ and briefly indicated as MSNA variability in the following. The choice of the duration of the time window (i.e., $5 \mathrm{~s}$ ) is the result of an optimization process. We computed the linear regression of the power of the variability of the MSNA burst rate in the frequency band centered about $0.1 \mathrm{~Hz}$ (from 0.04 to $0.15 \mathrm{~Hz}$ ), as assessed via autoregressive power spectral analysis (Pagani et al., 1997), on the sine of tilt table angle as a function of the window length. We tested window lengths of 3,5,10,15,20 s and we found out that the length of $5 \mathrm{~s}$ maximized the correlation coefficient of the linear regression (respectively $r=0.426, p=1.82 \times 10^{-3} ; r=0.441, p=1.19$ $\times 10^{-3} ; r=0.419, p=2.20 \times 10^{-3} ; r=0.245, p=8.35 \times$ $\left.10^{-2} ; r=0.384, p=5.39 \times 10^{-3}\right)$. Longer windows smoothed the 
T0
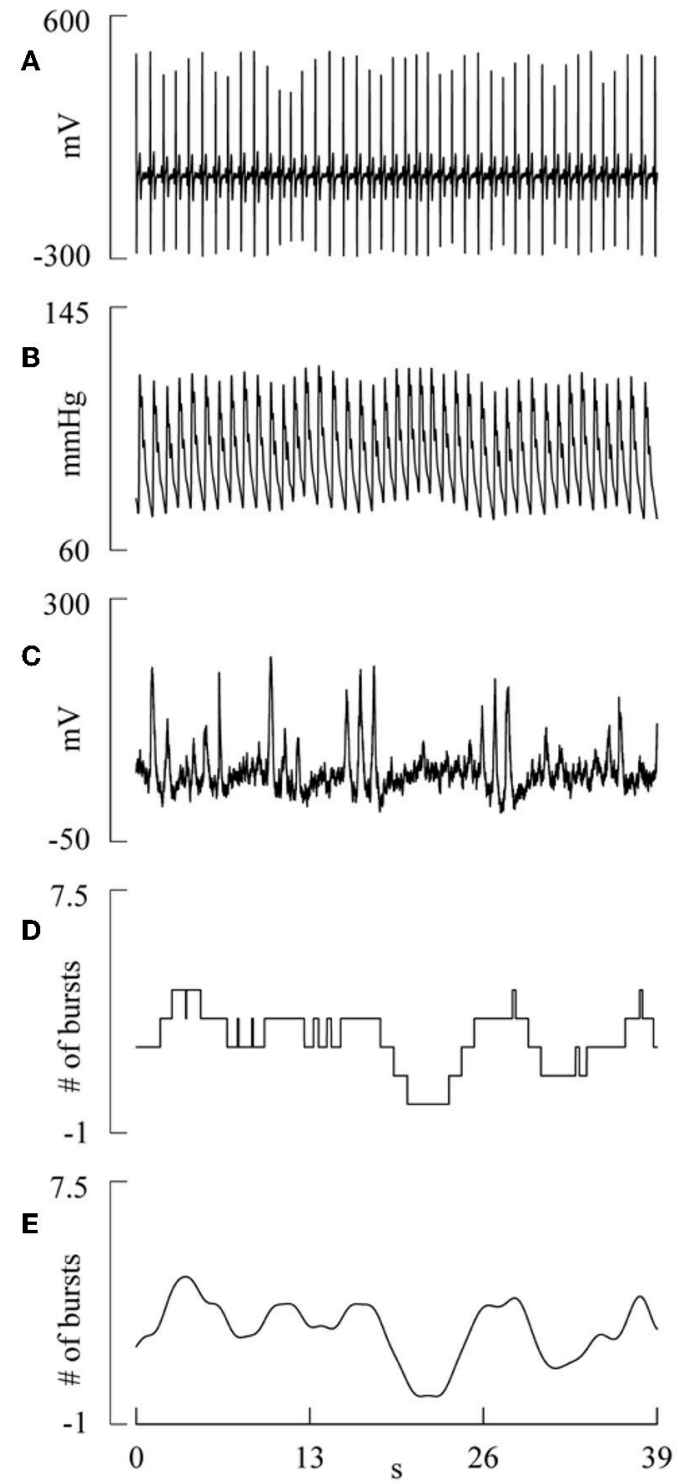

$\mathbf{F}$

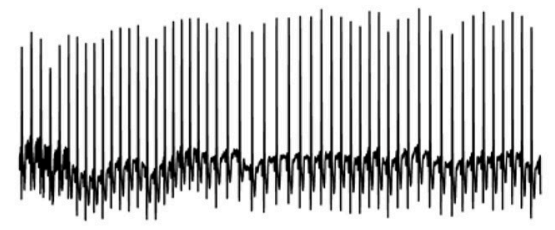

G

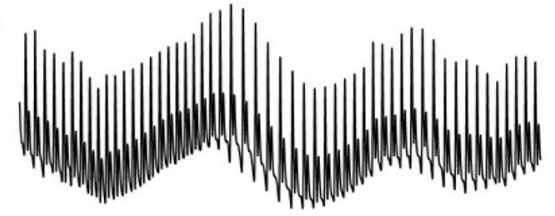

H

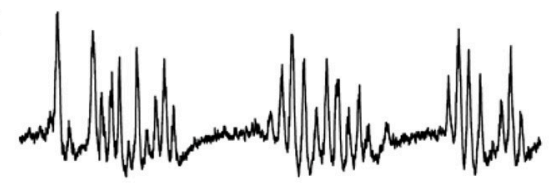

$\mathbf{I}$

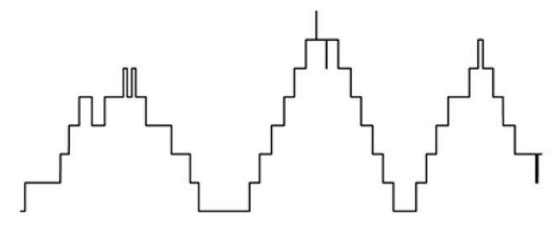

J

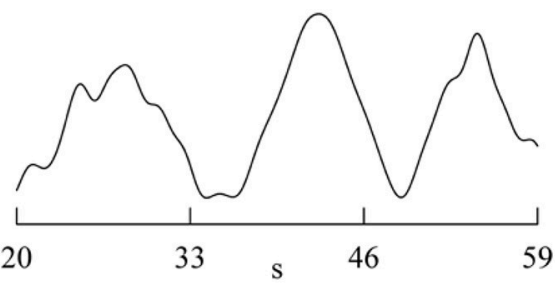

FIGURE 1 | The line plots show examples of ECG, invasive AP, integrated MSNA, step-wise burst-count MSNA and low-pass burst-count MSNA during T0 (A-E) and T60 (F-J). The slow oscillations in the low-pass burst-count MSNA occurring in phase opposition to slow changes of SAP and DAP visible on AP are more evident during T60 than T0, while faster oscillations are more present during T0.

oscillations about $0.1 \mathrm{~Hz}$ probably because it is highly likely that periods of MSNA silence were included in any window. Shorter windows (i.e., 3 beats) produced some smoothing as well, mainly as a consequence of the limited number of MSNA bursts detected in any window and the more important transitions from a MSNA burst rate level and its adjacent one compared to those relevant to longer window lengths.

Figure 2 provides a summary of the conventions used in this study for numbering the HP, SAP, DAP, and MSNA burst rate measurements. HP, SAP, DAP, and MSNA variability series of $N=256$ consecutive values were randomly selected within each experimental condition. If non-stationarities, such as very slow drifting of the mean or sudden changes of the variance, were evident despite the linear detrending, the random selection was repeated. Figure 3 shows examples of the beat-to-beat HP, SAP, DAP, and MSNA variability series derived from the same subject as in Figure 1 during T0 and T60. The mean and the variance of HP, SAP, DAP, and MSNA variability series were calculated, indicated as $\mu_{\mathrm{HP}}, \sigma_{\mathrm{HP}}^{2}, \mu_{S A P}, \sigma_{\mathrm{SAP}}^{2}$, $\mu_{\mathrm{DAP}}, \sigma_{\mathrm{DAP}}^{2}, \mu_{\mathrm{MSNA}}$, and $\sigma_{\mathrm{MSNA}}^{2}$, and expressed as $\mathrm{ms}, \mathrm{ms}^{2}$, $\mathrm{mmHg}, \mathrm{mmHg}^{2}, \mathrm{mmHg}, \mathrm{mmHg}^{2}$, bursts $\mathrm{s}^{-1}$, and bursts $\mathrm{s}^{2} \mathrm{~s}^{-2}$, respectively. 


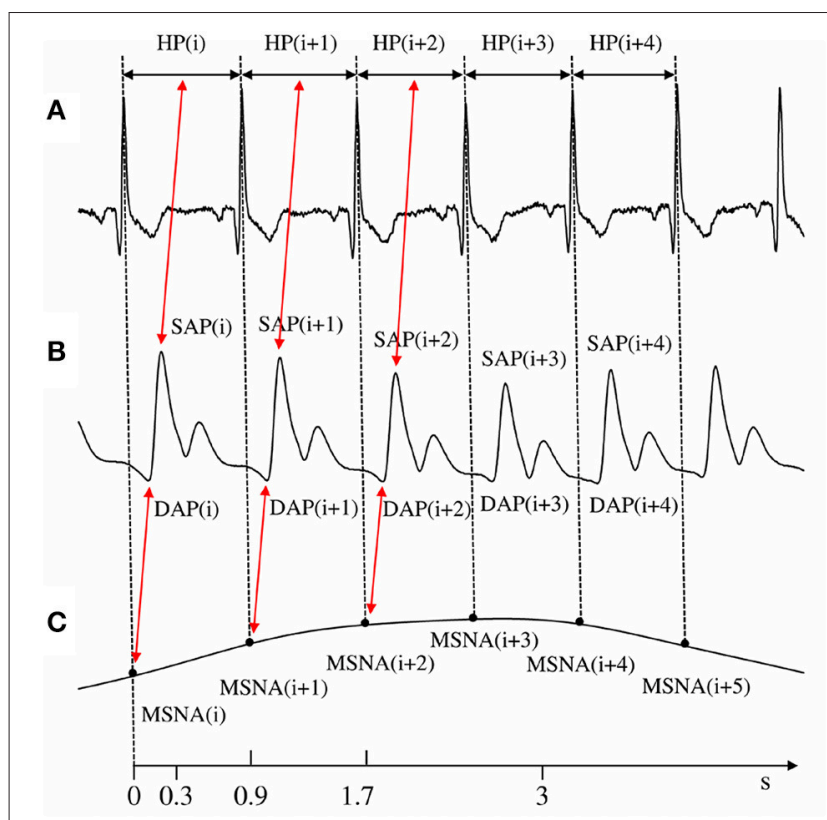

FIGURE 2 | The conventions for the construction of HP, SAP, DAP, and MSNA variability series from ECG (A), AP (B), and low-pass burst-count MSNA (C) are shown. The associations between HP and SAP values, exploited for the computation of $\mathrm{CBRS}_{\mathrm{SEQ}}$ and between MSNA variability and DAP utilized for the computation of SBRS $S_{S E Q}$, are reported as well. The time axis reports the lower and upper limits of the $\mathrm{CBR}$ and SBR latencies (i.e., 0.3-3.0 s and 0.9-1.7 s, respectively) assumed in this study.

\section{cBR Sequence Method}

cBRS estimation was based on cBR sequence method (Bertinieri et al., 1985) as implemented in Porta et al. (2000). This technique relies on the search for spontaneous sequences characterized by the contemporaneous increase or decrease of HP and SAP. The sequences are composed by three consecutive HPs and SAPs. A spontaneous $\mathrm{cBR}$ sequence was considered to be meaningful if it matched the following prerequisites (Laude et al., 2004): (1) the absolute value of the total HP variation was larger than $5 \mathrm{~ms}$ (i.e., $|\Delta \mathrm{HP}|>5 \mathrm{~ms}$ ); (2) the absolute value of the total SAP variation was larger than $1 \mathrm{mmHg}$ (i.e., $|\Delta \mathrm{SAP}|>1 \mathrm{mmHg}$ ); (3) the linear correlation coefficient computed in the $\left[\operatorname{SAP}(i), \mathrm{HP}\left(i+\tau_{\mathrm{HP}-\mathrm{SAP}}\right)\right]$ plane over a given $\mathrm{cBR}$ sequence, $\mathrm{r}_{\mathrm{HP}-\mathrm{SAP}}$, was larger than 0.85 , where $\tau_{H P-S A P}$ represents the lag between HP and SAP. Two alternative methods to fix $\tau_{\mathrm{HP}-\mathrm{SAP}}$ were tested: (i) $\tau_{\mathrm{HP}-\mathrm{SAP}}=0$ beats (Figure 2) to pick up the fast vagal arm of the cBR capable of modifying HP in reaction of SAP changes occurring within the same HP (Eckberg, 1976; Baselli et al., 1994); (ii) $\tau_{\text {HP-SAP was }}$ optimized in the set $\{0,1,2,3\}$ in keeping with the maximum of the HP-SAP cross-correlation function. The $\tau_{\text {HP-SAP }}$ optimization range was selected according to the minimum and maximum cBR latency set to 0.3 and 3.0 s, respectively (Eckberg, 1976; Porta et al., 2011): indeed, the association between $\operatorname{SAP}(i)$ and $\mathrm{HP}\left(\mathrm{i}+\tau_{\mathrm{HP}-\mathrm{SAP}}\right)$ due to $\mathrm{CBR}$ should be negligibly present with $\tau_{\text {HP-SAP }}>3$ (Figure 2). The slope of the regression line was calculated for each $\mathrm{cBR}$ sequence and subsequently averaged over all sequences. This average was taken as an estimate of
cBRS, labeled as $\mathrm{cBRS}_{\mathrm{SEQ}}$ and expressed in $\mathrm{ms}^{\cdot} \mathrm{mmHg}^{-1}$. The percentage of $\mathrm{cBR}$ sequences with respect to all sequences was computed as well and indicated as SEQ $\%_{c B R}$. SEQ $\%_{c B R}$ ranges between zero and 100 where zero indicates the absence of cBR sequences, while 100 indicates that all possible HP-SAP sequences are of $\mathrm{cBR}$ origin. While $\mathrm{CBRS}_{\mathrm{SEQ}}$ is taken as a measure of the $\mathrm{cBR}$ effectiveness, $\mathrm{SEQ} \%_{\mathrm{CBR}}$ is taken as a measure of the degree of $\mathrm{cBR}$ involvement.

\section{sBR Sequence Method}

sBRS $_{\text {SEQ }}$ was estimated with the same logic as $c B R S_{S E Q}$ over the MSNA and DAP beat-to-beat variability series. This application was made possible by the exploitation of the MSNA series defined in Marchi et al. (2016) expressing the MSNA variability in bursts $\mathrm{s}^{-1}$. Specifically, the sBR sequence technique relied on the search for spontaneous sequences characterized by the contemporaneous increase of MSNA burst rate and decrease of DAP or vice versa. The prerequisites relevant to linear correlation coefficient in the $\left[\mathrm{DAP}(i), \operatorname{MSNA}\left(i+\tau_{\mathrm{MSNA}-\mathrm{DAP}}\right)\right]$ plane, $\mathrm{r}_{\mathrm{MSNA}-\mathrm{DAP}}$, and the total variation of DAP, $|\triangle \mathrm{DAP}|$, reflected the usual setting of the $\mathrm{cBR}$ sequence analysis (i.e., $\left.\left|\mathrm{r}_{\text {MSNA-DAP }}\right|>0.85\right)$ and the typical bin size set in more traditional methods for sBRS estimation from spontaneous DAP variability (i.e., $|\triangle \mathrm{DAP}|>1 \mathrm{mmHg}$; Hart et al., 2010). Also the length of the sBR sequences was maintained equal to that of $\mathrm{cBR}$ sequences and corresponded to three consecutive DAPs and MSNA burst rate values. $\tau_{\text {MSNA-DAP, representing the lag }}$ between MSNA burst rate and DAP expressed in beats, was optimized in the set $\{0,1,2,3\}$ to fully account for the latency of sBR in modifying MSNA burst rate after sensing DAP (Kienbaum et al., 2001; Diedrich et al., 2009; Figure 2). The selected range of $\tau_{\text {MSNA-DAP }}$ is in keeping with that selected for cBR characterization and sBR latency. The prerequisite relevant to the absolute value of the MSNA burst rate variation, $|\triangle \mathrm{MSNA}|$, was optimized as well. We tested different minimal levels of $|\triangle \mathrm{MSNA}|$ corresponding to the prerequisite of $|\triangle \mathrm{MSNA}|>$ $x$ bursts $\mathrm{s}^{-1}$, where $x$ was initially set to 0.0 bursts $\mathrm{s}^{-1}$ and, then, increased in steps of 0.05 bursts $\mathrm{s}^{-1}$ up to 0.5 bursts $\mathrm{s}^{-1}$. The upper limit of 0.5 bursts $^{-1}$ was chosen according to the maximum $|\triangle \mathrm{MSNA}|$ observed in our protocol given the adopted sequence length. The slope of the regression line was calculated for each sBR sequence and subsequently averaged over all sequences. This average was taken as an estimate of sBRS, labeled as sBRS $\mathrm{SEQ}$ and expressed in bursts $\mathrm{s}^{-1} \cdot \mathrm{mmHg}^{-1}$. The percentage of $s B R$ sequences with respect to all sequences was computed as well and indicated as SEQ\% ${ }_{\mathrm{sBR}}$. SEQ\% $\%_{\mathrm{sBR}}$ ranges between zero and 100 where zero indicates the absence of sBR sequences, while 100 indicates that all possible MSNA-DAP sequences are of baroreflex origin. While $s B R S_{S E Q}$ is taken as a measure of the sBR effectiveness, $\mathrm{SEQ} \%_{\mathrm{SBR}}$ is taken as a measure of the degree of sBR involvement.

\section{BTA Method}

BTA is based on the computation of the percentage of MSNA bursts associated with a given level of DAP (Sundlöf and Wallin, 1978; Kienbaum et al., 2001; Hart et al., 2010). The size of the DAP bin was fixed to $1 \mathrm{mmHg}$ (Hart et al., 2010). 
T0

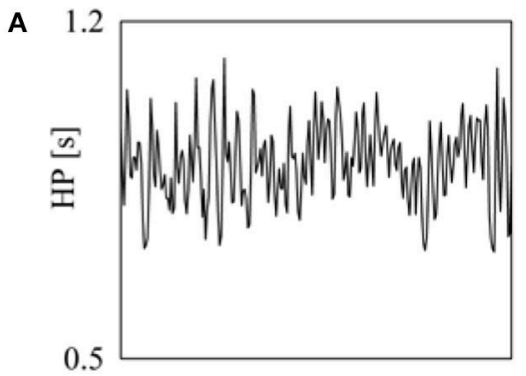

B

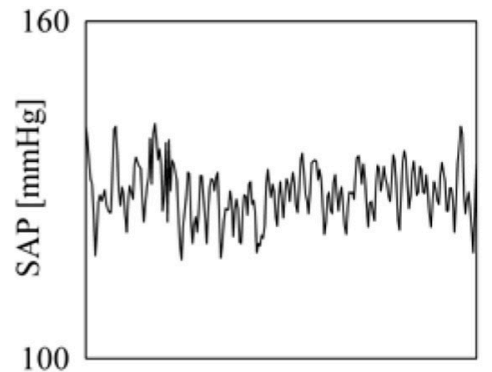

C
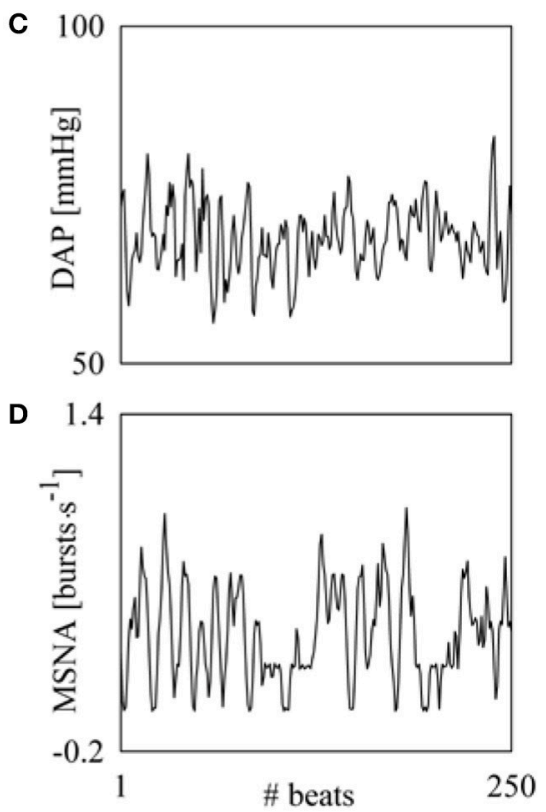

T60

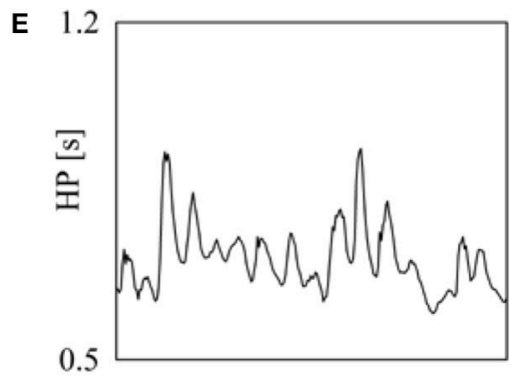

F 16

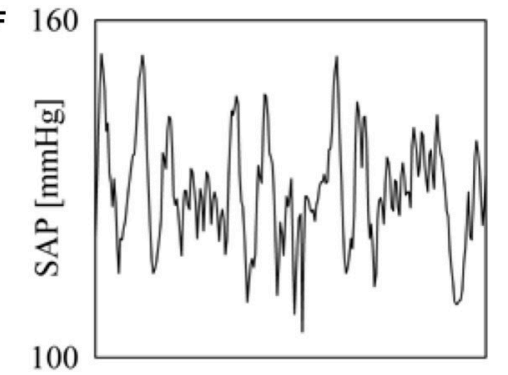

G
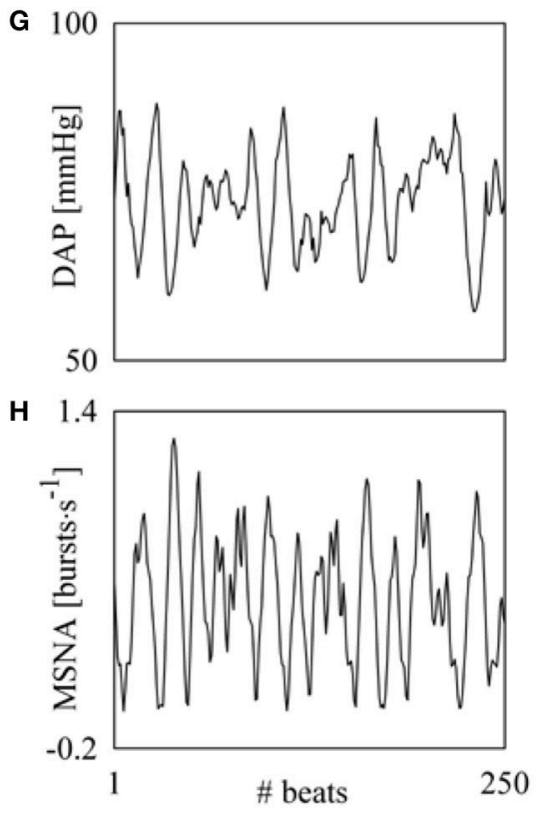

FIGURE 3 | The line plots show examples of HP, SAP, DAP, and MSNA beat-to-beat variability recorded during T0 (A-D) and T60 (E-H). Fast dynamics at the respiratory rate are more visible in HP and SAP series during TO (A,B) and in SAP during T60 (F). DAP and MSNA series (C,D,G,H) are dominated by rhythms at frequency slower than respiration.

More specifically, the percentage of heart beats associated to a given MSNA burst was plotted as a function of the mean DAP in the assigned bin and the slope of the regression line in this plane was taken as an estimate of the sBRS and indicated as sBRS $S_{B T A}$ in the following. To account for the sBR latency the MSNA burst was searched for in a time window ranging from 0.9 to $1.7 \mathrm{~s}$ starting from the R-wave peak (Kienbaum et al., 2001; Diedrich et al., 2009). Since the likelihood to find a MSNA bust decreases with DAP, sBRS $\mathrm{BTA}_{\mathrm{B}}$ is smaller than zero.

\section{Statistical Analysis}

We carried out the Bartlett test, or the Levene test when appropriate, to check for homoscedasticity of the computed parameters in the various experimental sessions. If the null hypothesis of homogeneity of variance was rejected, we logtransformed the absolute value of the parameter and the test for homoscedasticity was repeated. One-way analysis of variance (Dunnett's test for multiple comparisons), or Kruskal-Wallis one-way analysis of variance on ranks when appropriate (Dunn's 
test for multiple comparisons), was applied to test the significance of the changes of time domain parameters compared to T0. Paired $t$-test, or Wilcoxon signed rank test when appropriate, was applied to check the difference between SEQ\% ${ }_{\mathrm{cBR}}$ and SEQ\% ${ }_{\mathrm{sBR}}$ when data were pooled together regardless of the experimental condition. Linear regression analysis was carried out to check the presence of a linear relation of $\mathrm{CBRS}_{\mathrm{SEQ}}, \mathrm{SEQ} \%_{\mathrm{cBR}}, \mathrm{sBRS} \mathrm{SEQ}_{\mathrm{S}}$, and $S E Q \%{ }_{S B R}$ on the sine of the tilt table angles (the sine is taken because the stimulus is proportional to the component of the gravity parallel to the tilt table). The Pearson product moment correlation coefficient, $r$, was computed and the null hypothesis of a slope equal to zero (i.e., no linear relationship) was tested. If the null hypothesis of homoscedasticity was rejected, the result of linear regression analysis over the original values was checked after the log-transformation of the absolute values of the parameter. In the case of $s B R S_{S E Q}$ and SEQ\% ${ }_{s B R}$ the linear regression analysis was carried out as a function of the minimal $|\triangle \mathrm{MSNA}|$ utilized to detect a meaningful sBR sequence and $\tau_{\text {MSNA-DAP. Linear regression analysis of } s B R S_{B T A} \text { on } s B R S_{S E Q}}$ was performed to measure the degree of association between the nonpharmacological traditional method and the proposed method. A similar analysis was carried out between $\mathrm{sBRS}_{\mathrm{SEQ}}$ and $\mathrm{CBRS}_{\mathrm{SEQ}}$ and between SEQ\% ${ }_{\mathrm{SBR}}$ and SEQ\% ${ }_{\mathrm{cBR}}$ to assess the degree of association between $\mathrm{cBR}$ and $\mathrm{sBR}$ parameters. The Pearson product moment correlation coefficient, $r$, was computed and the null hypothesis of a slope equal to zero (i.e., no linear relationship) was tested after pooling together all subjects regardless of the experimental condition. If the null hypothesis of homoscedasticity was rejected of either of the variables or both, the result of linear regression analysis was checked after log-transforming the absolute values of the variable that did not pass the test for homogeneity of variance. Statistical analysis was carried out using a commercial statistical program (Sigmaplot, Systat Software, Inc, Chicago, IL, USA, ver.11.0). A $p<0.05$ was considered statistically significant.

\section{RESULTS}

\section{Optimization of the Parameters for Spontaneous sBR Sequence Analysis}

While the minimal $|\triangle \mathrm{DAP}|$, minimal $\left|\mathrm{r}_{\mathrm{MSNA}-\mathrm{DAP}}\right|$, and duration of the sBR sequence were set to $|\triangle \mathrm{DAP}|=1 \mathrm{mmHg},\left|\mathrm{r}_{\text {MSNA-DAP }}\right|$ $=0.85$, and 3 beats respectively, the minimal $|\triangle \mathrm{MSNA}|$ and $\tau_{\text {MSNA-DAP }}$ were optimized based on the analysis of the degree of linear association of $S E Q \%_{S B R}$ and ${ }_{s B R S} S_{S E Q}$ on tilt table inclination. The optimization of $|\triangle \mathrm{MSNA}|$ and $\tau_{\mathrm{MSNA}-\mathrm{DAP}}$ is based on the assumption that the best setting of SEQ\% ${ }_{S B R}$ and sBRS $\mathrm{SEQ}$ should allow the detection of both a positive linear relation of SEQ\% ${ }_{\mathrm{SBR}}$ on the relevance of the baroreflex unloading, taken as an indication of the progressive solicitation of sBR with the magnitude of the challenge, and a positive linear relation of $\mathrm{SBRS}_{\mathrm{SEQ}}$ with the sine of the tilt table angles, taken as a suggestion of a reduced effectiveness of sBR with the relevance of the stimulus. The correlation coefficients of the linear regression analysis of $\mathrm{SEQ} \%_{\mathrm{sBR}}$ on the sine of the tilt table inclination as a function of the minimal $\mid \triangle$ MSNA $\mid$ and $\tau_{\text {MSNA-DAP }}$ are reported in Table 1. Results indicate that, regardless of the value of $\tau_{\mathrm{MSNA}-\mathrm{DAP}}, \mathrm{SEQ} \%_{\mathrm{SBR}}$ was significantly and positively related to the magnitude of the orthostatic challenge at the smallest values of the minimal $\mid \triangle$ MSNA| (i.e., below 0.4 bursts $\mathrm{s}^{-1}$ ), thus suggesting the progressive sBR solicitation and the baroreflex origin of the detected sequences. The relation with the sine of the tilt table inclination disappeared at the highest values of the minimal $|\triangle \mathrm{MSNA}|$ because the more restrictive prerequisite reduced the number of spontaneous sBR sequences to such a level that prevented the detection of a significant relation of SEQ\% ${ }_{\mathrm{sBR}}$ to tilt table angles. The correlation coefficients of the linear regression analysis of $s B R S_{S E Q}$ on the sine of the tilt table inclination as a function of the minimal $|\triangle \mathrm{MSNA}|$ and $\tau_{\text {MSNA-DAP }}$ are reported in Table 2. sBRS $S_{\text {SEQ }}$ exhibited a significant and positive association with the magnitude of the orthostatic challenge only at $\tau_{\mathrm{MSNA}-\mathrm{DAP}}=0$ beats and at the smallest values of the minimal $|\triangle \mathrm{MSNA}|$ (i.e., below 0.15 bursts $\mathrm{s}^{-1}$ ). Taking together the results shown in Tables 1, 2, a significant positive association of both $\mathrm{SEQ} \%_{\mathrm{SBR}}$ and $\mathrm{sBRS} \mathrm{SEQ}_{\mathrm{S}}$ with the relevance of the orthostatic stimulus was observed at $\tau_{\text {MSNA-DAP }}=0$ beats and correlation coefficient picked

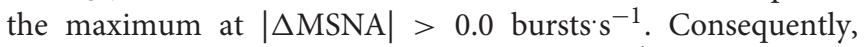
$\tau_{\text {MSNA-DAP }}=0$ and $\mid \Delta$ MSNA $\mid>0.0$ bursts $\mathrm{s}^{-1}$ were chosen as the most suitable setting for spontaneous sBR sequence analysis. The setting $\tau_{\text {MSNA-DAP }}=0$ was tested a posteriori by optimizing $\tau_{\text {MSNA-DAP }}$ in the set $\{0,1,2,3\}$ according to the minimum of the MSNA-DAP cross-correlation function: the minimum was found at $\tau_{\text {MSNA-DAP }}=0$ in $86 \%$ of the analyses.

\section{Results of Time Domain Indexes during Incremental Head-Up Tilt}

Time domain parameters computed over HP, SAP, DAP, and MSNA variability were reported in Table 3. As a function of tilt table inclination, $\mu_{\mathrm{HP}}$ gradually decreased showing a significant difference at T40 and T60 compared to T0. $\sigma_{\mathrm{HP}}^{2}, \mu_{\mathrm{SAP}}$ and $\mu_{\mathrm{DAP}}$ remained stable, while $\sigma_{\text {SAP }}^{2}$ and $\sigma_{\text {DAP }}^{2}$ significantly increased during T60. We found that both $\mu_{\mathrm{MSNA}}$ and $\sigma_{\mathrm{MSNA}}^{2}$ increased with the magnitude of the postural challenge, thus suggesting an increase of tonic MSNA and its modulation about the mean value. Indeed, while $\mu_{\mathrm{MSNA}}$ increased significantly during T40 compared to baseline, $\sigma_{\mathrm{MSNA}}^{2}$ rose significantly during both T40 and T60.

\section{cBR and sBR Controls during Incremental Head-Up Tilt}

Figure 4 shows examples of $\mathrm{cBR}$ and $\mathrm{sBR}$ sequence analyses carried out in a subject during T0 (Figures 4A,C) and T60 (Figures 4B,D). The panels show the linearly-interpolated $\mathrm{cBR}$ and $\mathrm{sBR}$ sequences whose mean slope is the $c B R S_{\mathrm{SEQ}}$ (Figures 4A,B) and sBRS $\mathrm{SEQ}$ (Figures 4C,D). The slopes of the $\mathrm{CBR}$ sequences are positive (Figures $4 \mathrm{~A}, \mathrm{~B}$ ), while the slopes of the sBR sequences are negative (Figures 4C,D). During T60 (Figure 4B) the $c B R$ sequences are flatter (i.e., the slopes are closer to zero) than during T0 (Figure 4A) and their number is larger. Since the analysis during T0 was carried out over series of the same length (i.e., 256 consecutive values) as during 
TABLE 1 | Correlation coefficient $r$ of the regression line of SEQ\% ${ }_{S B R}$ on the sine of the tilt table angles as a function of the minimal $|\triangle M S N A|$ and TMSNA-DAP.

\begin{tabular}{|c|c|c|c|c|}
\hline & $\tau_{\mathrm{MSNA} \text {-DAP }}=0$ beats & $\tau_{\text {MSNA-DAP }}=1$ beats & $\tau_{\text {MSNA-DAP }}=2$ beats & $\tau_{\text {MSNA-DAP }}=3$ beats \\
\hline$|\Delta \mathrm{MSNA}|>0.00$ bursts. $\mathrm{s}^{-1}$ & $0.468^{\star}$ & $0.482^{*}$ & $0.464^{*}$ & $0.521^{*}$ \\
\hline$|\Delta \mathrm{MSNA}|>0.05$ bursts. $\mathrm{s}^{-1}$ & $0.467^{\star}$ & $0.479^{*}$ & $0.466^{\star}$ & $0.520^{*}$ \\
\hline$|\Delta \mathrm{MSNA}|>0.15$ bursts. $\mathrm{s}^{-1}$ & $0.460^{*}$ & $0.464^{*}$ & $0.453^{\star}$ & $0.494^{*}$ \\
\hline$|\Delta \mathrm{MSNA}|>0.20$ bursts. $\mathrm{s}^{-1}$ & $0.413^{\star}$ & $0.441^{*}$ & $0.436^{\star}$ & $0.466^{\star}$ \\
\hline$|\Delta \mathrm{MSNA}|>0.35$ bursts. $\mathrm{s}^{-1}$ & $0.293^{\star}$ & $0.293^{*}$ & $0.294^{*}$ & $0.331^{*}$ \\
\hline$|\Delta \mathrm{MSNA}|>0.40$ bursts. $\mathrm{s}^{-1}$ & 0.258 & 0.267 & 0.278 & 0.281 \\
\hline$|\Delta \mathrm{MSNA}|>0.45$ bursts. $\mathrm{s}^{-1}$ & 0.250 & 0.289 & 0.259 & 0.311 \\
\hline$|\Delta \mathrm{MSNA}|>0.50$ bursts. $\mathrm{s}^{-1}$ & 0.275 & 0.266 & 0.269 & 0.296 \\
\hline
\end{tabular}

SBR, sympathetic baroreflex; $S E Q \%_{S B R}$, percentage of SBR sequences; $|\triangle M S N A|$, absolute value of the total MSNA burst rate variation during a spontaneous sBR sequence; $\tau_{M S N A-D A P}$, lag between MSNA and DAP. The symbol * indicates a $p<0.05$.

TABLE 2 | Correlation coefficient $r$ of the regression line of sBRS SEQ $_{\text {on }}$ the sine of the tilt table angles as a function of the minimal $|\Delta M S N A|$ and $\tau_{\text {MSNA-DAP. }}$

\begin{tabular}{|c|c|c|c|c|}
\hline & $\tau_{\text {MSNA-DAP }}=0$ beats & $\tau_{\mathrm{MSNA} \text {-DAP }}=1$ beats & $\tau_{\text {MSNA-DAP }}=2$ beats & $\tau_{\mathrm{MSNA}-\mathrm{DAP}}=3$ beats \\
\hline$|\Delta \mathrm{MSNA}|>0.00$ bursts. $\mathrm{s}^{-1}$ & $0.302^{*}$ & 0.026 & 0.123 & 0.161 \\
\hline$|\Delta \mathrm{MSNA}|>0.05$ bursts. $\mathrm{s}^{-1}$ & $0.307^{\star}$ & 0.015 & 0.128 & 0.160 \\
\hline$|\Delta \mathrm{MSNA}|>0.15$ bursts. $\mathrm{s}^{-1}$ & 0.274 & -0.030 & 0.100 & 0.147 \\
\hline$|\Delta \mathrm{MSNA}|>0.20$ bursts. $\mathrm{s}^{-1}$ & 0.238 & -0.038 & 0.116 & 0.127 \\
\hline$|\Delta \mathrm{MSNA}|>0.35$ bursts. $\mathrm{s}^{-1}$ & 0.212 & 0.020 & 0.138 & 0.227 \\
\hline$|\Delta \mathrm{MSNA}|>0.40$ bursts. $\mathrm{s}^{-1}$ & 0.201 & -0.029 & 0.126 & 0.263 \\
\hline$|\Delta \mathrm{MSNA}|>0.45$ bursts. $\mathrm{s}^{-1}$ & 0.275 & 0.084 & 0.230 & 0.278 \\
\hline$|\Delta \mathrm{MSNA}|>0.50$ bursts. $\mathrm{s}^{-1}$ & 0.269 & 0.184 & 0.227 & 0.272 \\
\hline
\end{tabular}

SBR, sympathetic baroreflex; SBRS SEQ, SBR sensitivity computed with the sBR sequence method; $|\triangle M S N A|$, absolute value of the total MSNA burst rate variation during a spontaneous sBR sequence; $\tau_{M S N A-D A P}$ lag between MSNA and DAP. The symbol * indicates a $p<0.05$.

TABLE 3 | Time domain parameters.

\begin{tabular}{|c|c|c|c|c|c|}
\hline Index & TO & T20 & T30 & $T 40$ & $T 60$ \\
\hline$\mu_{\mathrm{HP}}[\mathrm{ms}]$ & $1003 \pm 147$ & $927 \pm 137$ & $885 \pm 129$ & $806 \pm 113^{\star}$ & $688 \pm 120^{*}$ \\
\hline$\sigma_{\mathrm{HP}}^{2}\left[\mathrm{~ms}^{2}\right]$ & $5442 \pm 3440$ & $4553 \pm 3652$ & $4346 \pm 2605$ & $3005 \pm 1817$ & $2498 \pm 939$ \\
\hline$\mu_{\mathrm{SAP}}[\mathrm{mmHg}]$ & $122.8 \pm 21.4$ & $123.0 \pm 17.9$ & $125.2 \pm 20.4$ & $124.2 \pm 15.6$ & $122.3 \pm 16.7$ \\
\hline$\sigma_{\text {SAP }}^{2}\left[\mathrm{mmHg}^{2}\right]$ & $13.7 \pm 9.2$ & $11.8 \pm 6.8$ & $14.8 \pm 8.9$ & $21.9 \pm 14.2$ & $35.5 \pm 22.2^{*}$ \\
\hline$\mu_{\mathrm{DAP}}[\mathrm{mmHg}]$ & $67.6 \pm 11.4$ & $68.6 \pm 11.1$ & $70.0 \pm 14.9$ & $72.9 \pm 11.3$ & $75.5 \pm 14.8$ \\
\hline$\sigma_{\text {MSNA }}^{2}\left[\right.$ bursts $\left.^{2} \cdot \mathrm{s}^{-2}\right]$ & $0.033 \pm 0.010$ & $0.043 \pm 0.015$ & $0.044 \pm 0.008$ & $0.057 \pm 0.018^{\star}$ & $0.055 \pm 0.021^{*}$ \\
\hline
\end{tabular}

$\mu_{H P}, H P$ mean; $\sigma_{H P}^{2}$, HP variance; $\mu_{S A P}$, SAP mean; $\sigma_{S A P}^{2}$, SAP variance; $\mu_{D A P}, D A P$ mean; $\sigma_{D A P}^{2}$, DAP variance; $\mu_{M S N A}, M S N A$ burst rate mean; $\sigma_{M S N A}^{2}, M S N A$ burst rate variance; TO, T20, T30, T40, and T60, head-up tilt at 0, 20,30, 40, and 60 degrees. Values are expressed as mean \pm standard deviation. The symbol ${ }^{*}$ indicates a $p<0.05 \mathrm{vs}$. T0.

T60, also SEQ\% ${ }_{\mathrm{cBR}}$ was higher during T60 than T0. Indeed, $\mathrm{SEQ} \%_{\mathrm{CBR}}$ is 3.70 and 9.78 during $\mathrm{T} 0$ and T60, respectively and $\mathrm{CBRS}_{\mathrm{SEQ}}$ is 35.56 and $14.83 \mathrm{~ms}^{\mathrm{mmHg}} \mathrm{mm}^{-1}$. The comparison of the results relevant to sBR sequences during T60 (Figure 4D) and T0 (Figure 4C) leads to the same conclusion. Indeed, sBR sequences are less steep during T60 (i.e., sBRS $S_{S E Q}$ is -0.120 and -0.075 

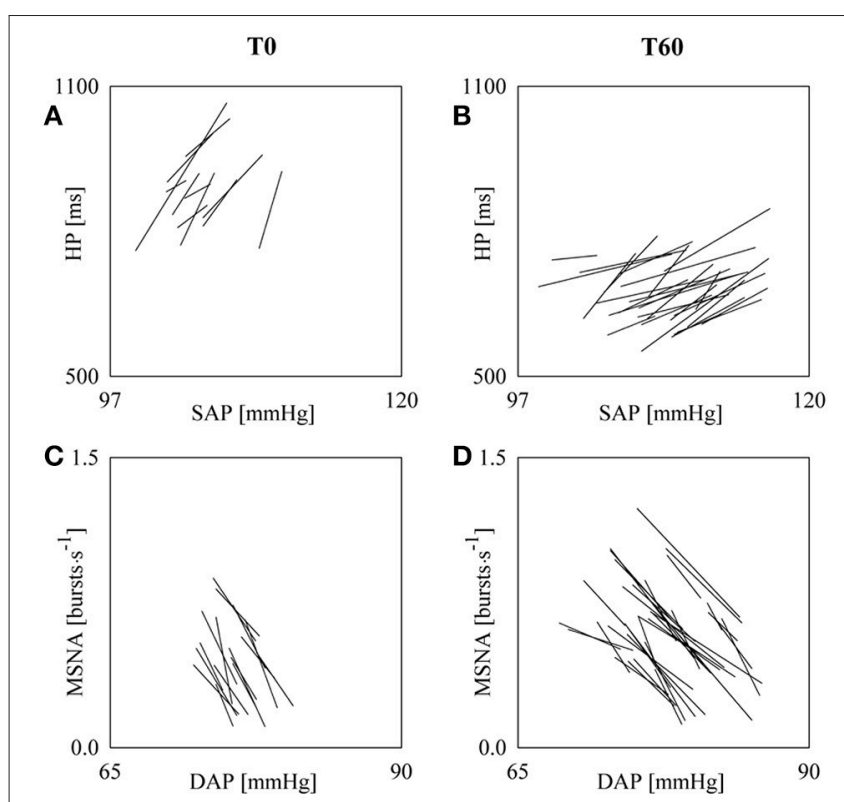

FIGURE 4 | The line plots show the linearly-interpolated spontaneous CBR $(A, B)$ and sBR $(C, D)$ sequences derived in a subject from SAP and HP series and from DAP and MSNA series respectively during TO $(A, C)$ and T60 (B,D). The analyses were carried out over SAP, HP, DAP, and MSNA series of 256 values. The larger number of $\mathrm{CBR}$ and SBR sequences, leading to larger SEQ\% ${ }_{\mathrm{CBR}}$ and $\mathrm{SEQ} \%_{\mathrm{SBR}}$, and the flatter slopes, leading to $\mathrm{CBRS} \mathrm{SEQ}_{\mathrm{S}}$ and SBRS $S_{S E Q}$ closer to zero, are evident during T60 compared to TO. bursts $\mathrm{s}^{-1} \cdot \mathrm{mmHg}^{-1}$ during $\mathrm{T} 0$ and $\mathrm{T} 60$, respectively) and their number increases (i.e., SEQ\% ${ }_{\mathrm{SBR}}$ is 5.39 and 12.68 during $\mathrm{T} 0$ and T60, respectively).

Figure 5 shows the individual values (open circles) of $c B R S_{S E Q}$ (Figure 5A) and $\mathrm{SEQ} \%_{\mathrm{CBR}}$ (Figure 5B) as a function of the sine of the tilt table inclination. These results were obtained with $\tau_{\mathrm{HP}-\mathrm{SAP}}=0$. The regression line of the variable on the sine of the tilt table angles (solid line) and its 95\% confidence interval (dotted lines) are plotted as well if the slope of the regression line is significantly larger than zero. A significant negative association of $\mathrm{cBRS}_{\mathrm{SEQ}}$ (Figure 5A, $r=-0.607, p=$ $\left.2.93 \times 10^{-6}\right)$ and a significantly positive correlation of SEQ\% ${ }_{\mathrm{CBR}}$ (Figure 5B, $r=0.328, p=2.02 \times 10^{-2}$ ) with the relevance of the postural challenge were found. A slightly stronger association was obtained after optimizing $\tau_{H P-S A P}$ on individual basis: indeed, $\mathrm{cBRS}_{\mathrm{SEQ}}$ was negatively associated with tilt table angles with $r=-0.687$ and $p=3.71 \times 10^{-8}$, while SEQ\% ${ }_{\mathrm{cBR}}$ was positively associated with $r=0.454, p=9.30 \times 10^{-4}$.

Figure 6 shows the individual values (open circles) of sBRS $S_{S E Q}$ (Figure 6A) and SEQ\% ${ }_{\mathrm{SBR}}$ (Figure 6B) as a function of the sine of the tilt table angle. According to the optimization procedure these results were obtained with $\tau_{\mathrm{MSNA}-\mathrm{DAP}}=0$ beats and $|\triangle \mathrm{MSNA}|>0.0$ bursts $^{-1}{ }^{-1}$. The regression line of the variable on the sine of the tilt table angles (solid line) and its 95\% confidence interval (dotted lines) are plotted as well if the slope of the regression line is significantly larger than zero. Significant

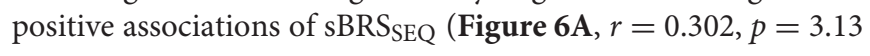

$\times 10^{-2}$ ) and SEQ\% ${ }_{\mathrm{sBR}}$ (Figure 6B, $r=0.468, p=5.38 \times 10^{-4}$ ) with the magnitude of the orthostatic challenge were found.

Figure 7 shows the percentage of sequences (SEQ\%) as a function of the arm of the baroreflex (i.e., cBR and sBR). SEQ\% values were pooled together regardless of the experimental condition. Values are reported as mean plus standard deviation. SEQ\% ${ }_{\mathrm{cBR}}$ was zero in one subjects during T0. SEQ\% ${ }_{\mathrm{cBR}}$ and SEQ\% ${ }_{S B R}$ were $4.77 \pm 3.99$ and $8.84 \pm 6.52$, respectively. SEQ\% ${ }_{s B R}$ was significantly higher than SEQ\% ${ }_{c B R}$.

Figure 8 shows the pairs (open circles) in the planes (sBRS $S_{S E Q}$, $\left.\mathrm{sBRS}_{\mathrm{BTA}}\right)$ as estimated from each subject regardless of the experimental condition. The regression line of $s B R S_{B T A}$ on sBRS $_{\text {SEQ }}$ (solid line) and its 95\% confidence interval (dotted lines) are plotted as well if the slope of the regression line is significantly larger than zero. A significant association between sBRS $S_{S E Q}$ and sBRS $_{\text {BTA }}\left(r=0.343, p=1.36 \times 10^{-2}\right)$ was detected.

Figure 9 shows the pairs (open circles) in the planes (cBRS ${ }_{\mathrm{SEQ}}$, $\mathrm{sBRS}_{\mathrm{SEQ}}$ ) (Figure 9A) and $\left(\mathrm{SEQ} \%_{\mathrm{cBR}}, \mathrm{SEQ} \%_{\mathrm{sBR}}\right)$ (Figure 9B) as estimated from each subject regardless of the experimental condition. The regression lines of $\mathrm{sBRS}_{\mathrm{SEQ}}$ on $\mathrm{CBRS}_{\mathrm{SEQ}}$ and SEQ $\%_{\text {sBR }}$ on SEQ $\%_{\text {CBR }}$ (solid line) and their $95 \%$ confidence interval (dotted lines) are plotted as well because both the slopes of the regression lines are significantly larger than zero. sBRS was significantly and negatively related to $\mathrm{cBRS}$ SEQ (Figure 9A, $\left.r=-0.302, p=3.30 \times 10^{-2}\right)$, while SEQ\% ${ }_{\mathrm{sBR}}$ was significantly and positively associated with SEQ\% ${ }_{\mathrm{CBR}}$ (Figure 9B, $r=0.340$, $p=1.57 \times 10^{-2}$ ).

\section{DISCUSSION}

The main findings of this study can be summarized as follows: (i) we proposed a spontaneous sBR sequence method exploiting a previously defined MSNA variability series describing the fluctuations of MSNA burst rate about its mean value; (ii) we utilized an incremental head-up tilt protocol to optimize the parameters necessary to detect $s B R$ sequences and we confirmed that the involvement of $s B R$ increased with tilt table angles, while its effectiveness decreased (i.e., SEQ\% ${ }_{s B R}$ increased and $s B R S_{S E Q}$ became less negative); (iii) in addition to the $s B R$ we characterized $c B R$ using the $c B R$ sequence method and we confirmed that the involvement of the $c B R$ increased with the magnitude of the orthostatic challenge and its effectiveness gradually declined (i.e., SEQ\% ${ }_{\mathrm{cBR}}$ increased and cBRS $_{\text {SEQ }}$ decreased toward zero); (iv) sBRS $_{\mathrm{SEQ}}$ was correlated with that computed via a more traditional static approach (i.e., BTA); (v) cBRS SEQ $_{\text {and }}$ sBRS $S_{\text {SEQ }}$ were significantly correlated as well as SEQ\% ${ }_{c B R}$ and SEQ\% ${ }_{s B R}$ even though both correlations were weak, thus suggesting that the $\mathrm{cBR}$ and sBR controls exhibit a certain degree of independence, while they are both activated in relation to the magnitude of the postural challenge.

\section{Spontaneous sBR Sequence Method}

Traditionally the sBR was assessed via the modified Oxford method (Rudas et al., 1999; Studinger et al., 2009; Dutoit et al., 2010; Hart et al., 2010). This pharmacological method imposes a rapid and wide modification of AP by administering in rapid succession a depressor agent (i.e., sodium nitroprusside) followed 

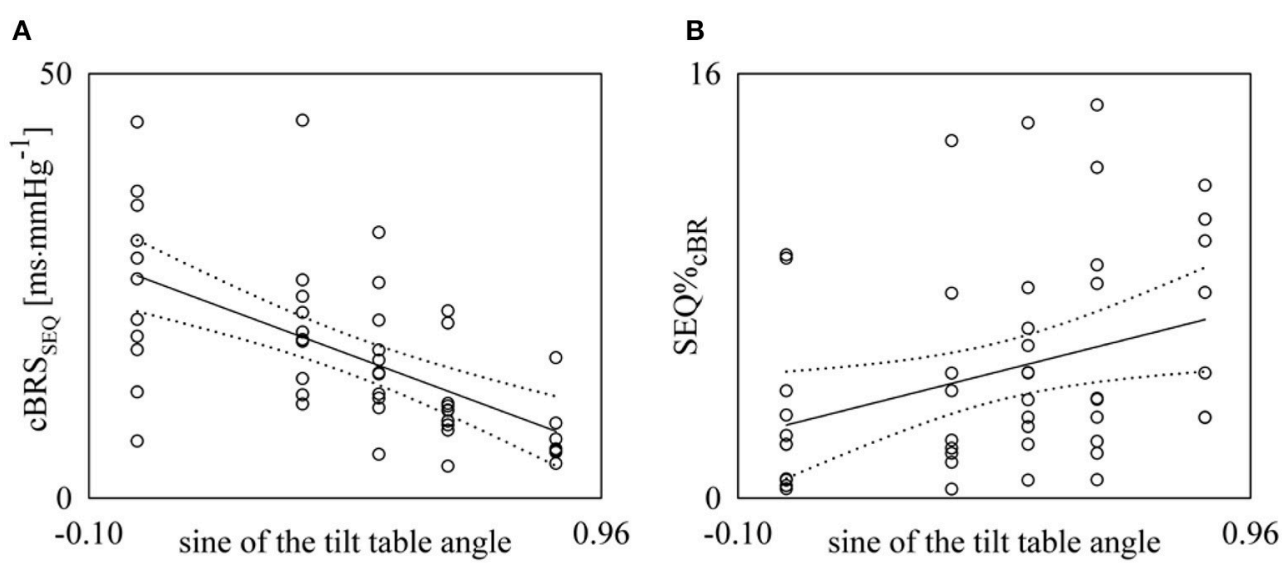

FIGURE 5 | Linear regression analyses of $c B R S_{\mathrm{SEQ}}(\mathrm{A})$ and $\mathrm{SEQ} \%_{\mathrm{CBR}}(\mathrm{B})$ on the sine of the tilt table angles during incremental orthostatic challenge. Individual values (open circles) of $\mathrm{CBRS} \mathrm{SEQ}_{\mathrm{S}}$ and SEQ\% ${ }_{\mathrm{CBR}}$ are shown as a function of the sine of the tilt table inclination. The linear regression (solid line) and its $95 \%$ confidence interval (dotted lines) are plotted as well if the slope of the regression line is significantly larger than zero with $p<0.05$.
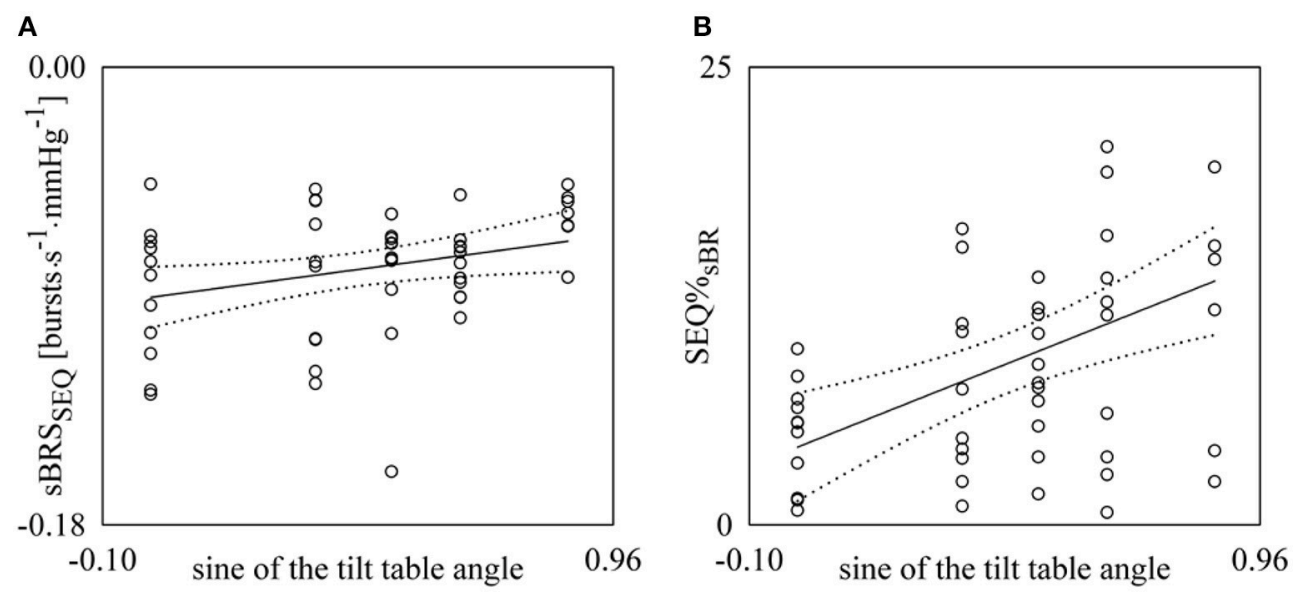

FIGURE 6 | Linear regression analyses of $\mathrm{SBRS}_{\mathrm{SEQ}}$ (A) and SEQ\% ${ }_{S B R}$ (B) on the sine of the tilt table angles during incremental orthostatic challenge. Individual values (open circles) of SBRS $S_{S E Q}$ and $\mathrm{SEQ} \%_{\mathrm{SBR}}$ are shown as a function of the sine of the tilt table inclination. The linear regression (solid line) and its $95 \%$ confidence interval (dotted lines) are plotted as well if the slope of the regression line is significantly larger than zero with $p<0.05$.

by a pressor one (i.e., phenylephrine). The modified Oxford method, originally devised for the characterization of the cBR (Ebert and Cowley, 1992), assesses sBR in nonphysiological conditions evoked by the administration of vasoactive drugs. In the attempt to overcome this issue, a static method based on spontaneous MSNA and DAP fluctuations has been devised (Sundlöf and Wallin, 1978; Kienbaum et al., 2001; O'Leary et al., 2003; Fu et al., 2006; Ichinose et al., 2006; Keller et al., 2006; Hart et al., 2010, 2011). The nonpharmacological method is grounded on counting the percentage of MSNA bursts associated with binned values of DAP observed during spontaneous fluctuations of AP and on the assessment of the linear relation describing the progressive inhibition of MSNA with DAP. In addition to the avoidance of the administration of the pharmacological stimulus, this method has an additional advantage: indeed, given the smallness of spontaneous physiological fluctuations of DAP, the hypothesis of linearity of the relation between AP and MSNA is more likely to be fulfilled compared to the pharmacological method. The potential drawback of the nonpharmacological method lies in its inability to assure the sole exploration of the causal link from DAP to MSNA. Indeed, since it is based on a static association of MSNA bursts with DAP values, the high MSNA-DAP correlation might not be the effect of dynamical variations of DAP (i.e., rises or falls) followed by the appropriate MSNA burst rate change compatible with a sBR origin (i.e., MSNA burst rate decreases or increases, respectively). Another potential drawback of the nonpharmacological approach is that it requires relatively long recordings to guarantee that a sufficient number of MSNA bursts can be associated with any bin of DAP.

In this study we propose a method exploiting the spontaneous fluctuations of MSNA burst rate and DAP about their mean values. The method is inspired by the $c B R$ sequence technique 


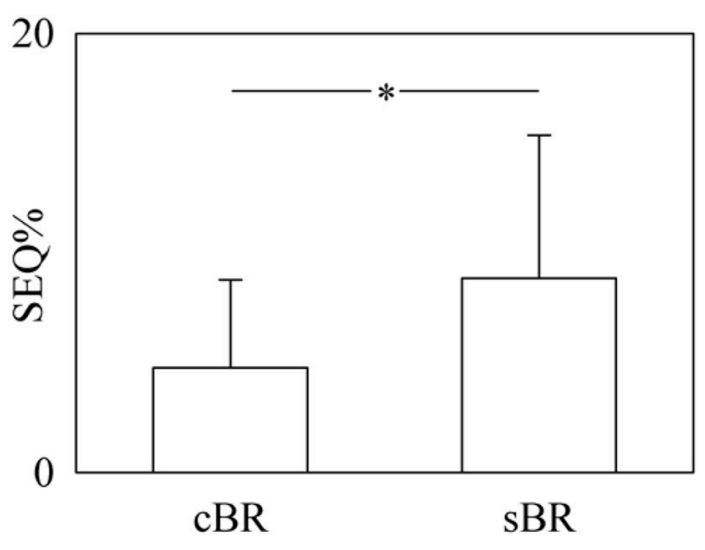

FIGURE 7 | The bar graph shows the percentage of sequences (SEQ\%) as a function of the arm of the baroreflex (i.e., cBR and sBR). SEQ\% values were pooled together regardless of the experimental condition. Values are given as mean plus standard deviation. The symbol * denotes a $p<0.05$

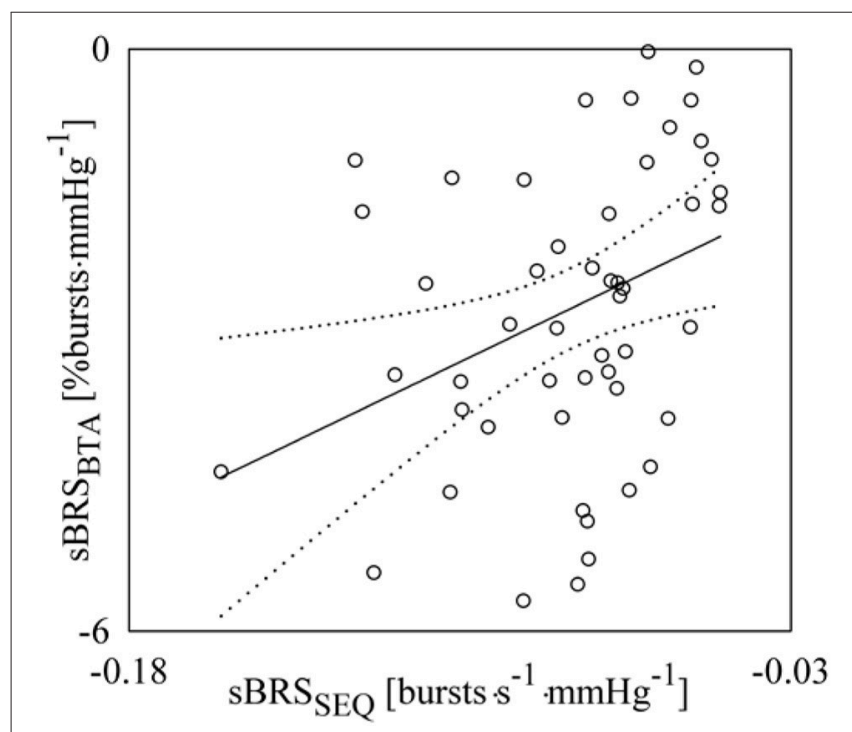

FIGURE 8 | Linear correlation analysis of sBRS BTA $_{\text {on }}$ sBRS SEQ $_{\text {during }}$ incremental orthostatic challenge. Individual pairs (open circles) in the plane (sBRS $S_{S E Q}$, SBRS $_{\mathrm{BTA}}$ ) are shown after pooling all subjects together regardless of the experimental condition. The linear regression (solid line) and its $95 \%$ confidence interval (dotted lines) are plotted as well if a significant association between the two sBRS measures was found with $p<0.05$.

frequently utilized to assess $\mathrm{CBR}$ via the detection of SAP and HP sequences featuring contemporaneous increases or decreases of both variables (Bertinieri et al., 1985). By analogy with the $c B R$ sequence technique, the sBR sequence method is based on the search of spontaneous sequences in MSNA and DAP series characterized by the contemporaneous decrease of DAP and increase of MSNA burst rate or vice versa. The sBR sequence method features two relevant advantages compared to the nonpharmacological methods (Sundlöf and Wallin, 1978; Kienbaum et al., 2001; Hart et al., 2010): (i) it is fully causal, because it is explicitly based on the detection of sequences that can be exclusively attributed to the sBR, thus avoiding the confounding factor related to the association of MSNA with DAP values when their variations are incompatible with a working sBR; (ii) since, in principle, it is sufficient to find a unique sBR sequence to compute the sBRS, this method can be applied to very short recordings of MSNA and DAP. The proposed approach was made possible by the exploitation of a recently defined MSNA variability quantifying the modulations of the MSNA burst rate about its mean (Marchi et al., 2016). The dimensionality of the recently proposed calibrated MSNA variability signal (i.e., bursts $\mathrm{s}^{-1}$ ) allows us to express sBRS with its natural units (i.e., bursts $\mathrm{s}^{-1} \mathrm{mmHg}^{-1}$ ), thus facilitating comparison among sBRS values derived from different experimental conditions and groups without requiring normalization procedures. This comparison would be more difficult if the more classical definition of MSNA variability based on low-pass filtering procedure of the integrated MSNA signal preserving the frequency content from 0.0 to $0.5 \mathrm{~Hz}$ was utilized (Saul et al., 1990; Pagani et al., 1997; Nakata et al., 1998; Taylor et al., 1998; Cooke et al., 1999; Furlan et al., 2000; Kamiya et al., 2005; Ryan et al., 2011). Indeed, the application of the proposed sBR sequence analysis to the low-pass filtered MSNA variability would lead to express sBRS in less natural units and would require some normalization procedures to avoid the dependence of sBRS on experimental factors such as position of the electrode, number of active units, amplification gain, and effects of noise superposed to the MSNA signal (Marchi et al., 2016). Normalization procedures are applied by traditional static methods for sBR characterization based on the association between amplitude or area of the MSNA burst and DAP value as well (Sundlöf and Wallin, 1978; Kienbaum et al., 2001). Even though the proposed method characterized sBR in the time domain, an alternative sBR characterization in the cardiac beat domain can be easily implemented by following this approach and expressing the MSNA variability in bursts per 100 beats.

\section{Optimization of the Parameters Utilized to Detect the Spontaneous sBR Sequences}

The minimal $|\triangle \mathrm{DAP}|$, being overcome to assure that the input was sufficiently exciting for activating the reflex, was set to $1 \mathrm{mmHg}$ in agreement with the minimal size of the bin utilized to quantize DAP in the nonpharmacological characterization of the sBR (Hart et al., 2010). In addition, this choice has the remarkable advantage of being the most frequent setting for the minimal $|\triangle \mathrm{SAP}|$ utilized by the cBR sequence analysis (Laude et al., 2004), thus allowing a more homogenous comparison between results derived from the proposed $\mathrm{sBR}$ sequence analysis and the traditional cBR sequence method (Bertinieri et al., 1985). The minimal $\left|r_{\text {MSNA-DAP }}\right|$, being overcome to assure that the degree of MSNA-DAP association was significant over a presumed sBR sequence, was set to 0.85 again in keeping with the similar setting in $\mathrm{CBR}$ sequence analysis. The length of the $\mathrm{sBR}$ sequence was set to 3 beats according to the minimal number of pairs in the plane $\left[\mathrm{DAP}(i), \operatorname{MSNA}\left(i+\tau_{\mathrm{MSNA}-\mathrm{DAP}}\right)\right]$ allowing a meaningful computation of a regression line. A similar setting was adopted 

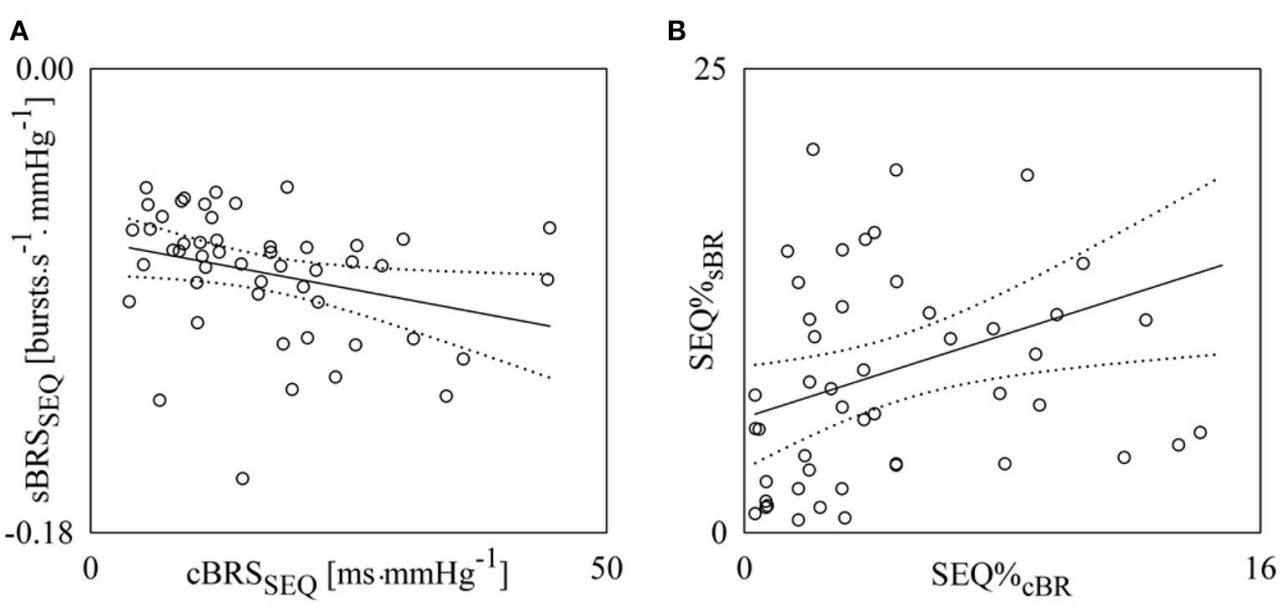

FIGURE 9 | Linear regression analyses of sBRS ${ }_{\mathrm{SEQ}}$ on cBRS ${ }_{\mathrm{SEQ}}(\mathrm{A})$ and $\mathrm{SEQ} \%_{\mathrm{SBR}}$ on $\mathrm{SEQ} \%_{\mathrm{CBR}}(\mathrm{B})$ during incremental orthostatic challenge.

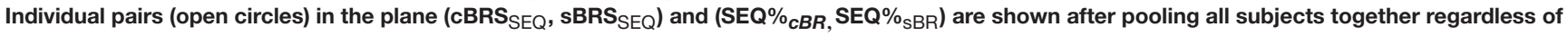
the experimental condition. The linear regression (solid line) and its $95 \%$ confidence interval (dotted lines) are plotted as well if the slope of the regression line is significantly larger than zero with $p<0.05$.

in cBR sequence analysis as well (Porta et al., 2000). While the minimal $|\triangle \mathrm{DAP}|$, the minimal $\left|\mathrm{r}_{\mathrm{MSNA}-\mathrm{DAP}}\right|$ and the length of the sBR sequence were chosen according to values present in literature with the main purpose to favor comparison, the minimal $|\triangle \mathrm{MSNA}|$, being overcome to assure that the magnitude of the $\mathrm{sBR}$ response to $|\triangle \mathrm{DAP}|$ was significant, and the latency

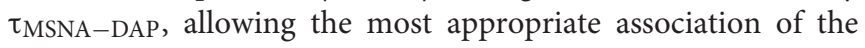
sensed AP variation with the MSNA burst rate change, were optimized. The parameters were chosen as the ones that allowed us to detect a gradually increasing involvement of sBR control in governing MSNA-DAP variability interactions and a progressive decrease of the effectiveness of the MSNA-DAP relation with the magnitude of the orthostatic stimulus as indicated by the positive relations of SEQ\% ${ }_{s B R}$ and $s B R S_{S E Q}$ with the sine of the tilt table angles. Both these relations are expected because they were found using a more traditional nonpharmacological static approach that does not require normalization (i.e., BTA) based on binning DAP values and finding the percentage of MSNA bursts associated with them (Ichinose et al., 2006; Barbic et al., 2015; Marchi et al., 2015). The activation of sBR during a postural challenge prevents the AP drop by inducing peripheral vasoconstriction ( $\mathrm{Fu}$ et al., 2006), while the reduction of sBRS was mainly attributed to the rise of DAP due to vasoconstriction (Ichinose et al., 2006). $\mid \Delta$ MSNA $\mid>0.0$ bursts $\mathrm{s}^{-1}$ and $\tau_{\mathrm{MSNA}-\mathrm{DAP}}=0$ beats allowed us to find both relationships to the magnitude of the orthostatic challenge with the strongest degree of association. The setting $|\triangle \mathrm{MSNA}|>0.0$ bursts $^{-1}{ }^{-1}$ indicates that there is no particular need to prevent the detection of meaningless variations of MSNA burst rate that, in association with the opposite sign variation of DAP, might produce spurious detections of sBR sequences. This setting might be the consequence of the exploited MSNA variability being inherently unaffected by noise because it is defined as the modulation of the MSNA burst rate about its mean value. We found that any increase of the minimal level of $|\triangle \mathrm{MSNA}|$ above 0.0 bursts $\mathrm{s}^{-1}$ leads to a drop in the number of the detected $\mathrm{sBR}$ sequences that reduces the degree of association of SEQ\% ${ }_{s B R}$ with the magnitude of the orthostatic challenge. The setting $\tau_{\text {MSNA-DAP }}=0$ beats suggests that the best association of $s B R S_{S E Q}$ and $S E Q \%{ }_{s B R}$ with tilt table angles was found when the sBR sequences were built by linking DAP to MSNA burst rate within the same beat (i.e., within a latency smaller than one HP). As shown in Figure 2 this result is compatible with the adopted latency of the sBR. Since the minimum of the MSNADAP cross-correlation function in the range of lags from 0 to 3 was found at lag zero in the majority of the analyses (i.e., $86 \%), \tau_{\text {MSNA-DAP }}=0$ appears to be reliable during the overall experimental protocol.

\section{sBR Sequence Method vs. BTA Approach}

BTA method estimates the linear relation of the percentage of MSNA bursts associated with a given binned DAP value on DAP values. This static approach is completely different from the dynamical technique here proposed. Indeed, the sBR sequence approach links a DAP change to a variation of the MSNA burst rate only if their signs are opposite, while in the BTA method MSNA burst occurrence and DAP values are associated regardless of whether variations of DAP produce the opposite changes in the probability of detecting a MSNA burst. Despite this structural difference between the sBR sequence method and BTA approach, we found that $s B R S_{S E Q}$ and $s B R S_{B T A}$ were significantly and positively correlated. This association might be the result of their common features: indeed, both the methods work on the rate of appearance of the MSNA burst and do not require normalization. However, the level of association is not strong, thus suggesting that the two sBRS estimates should not be considered interchangeable and, conversely, they should be regarded as describing different aspects of the same reflex. 


\section{Spontaneous cBR during Incremental Head-Up Tilt}

Graded head-up tilt induces a gradual sympathetic activation and vagal withdrawal occurring in response to baroreflex unloading associated to central hypovolemia (Montano et al., 1994; Cooke et al., 1999; Furlan et al., 2000; O'Leary et al., 2003; Kamiya et al., 2005; Fu et al., 2006; Porta et al., 2007; Lambert et al., 2008; Baumert et al., 2011; El-Hamad et al., 2015; Marchi et al., 2016; Porta et al., 2016). The modification of the state of the autonomic nervous system in response to head-up tilt with inclination lower than or equal to than $60^{\circ}$ produced a significant tachycardia with limited variations of AP (Bahjaoui-Bouhaddi et al., 2000; O'Leary et al., 2003; Fu et al., 2006; Porta et al., 2011). Our time domain parameters were in agreement with the classical characterization of cardiovascular variables during graded headup tilt: indeed, the increase of $\mu_{\mathrm{MSNA}}$ indicates a tendency toward a tonic sympathetic activation leading to a progressive reduction of $\mu_{\mathrm{HP}}$, while $\mu_{\mathrm{SAP}}$ and $\mu_{\mathrm{DAP}}$ were preserved. The progressive increase of $\sigma_{\text {MSNA }}^{2}$ with the tilt table inclination indicates that the magnitude of the fluctuations of the MNSA burst rate about its mean value increased and this rise is likely to be responsible for the increase of $\sigma_{\text {SAP }}^{2}$ and $\sigma_{\text {DAP }}^{2}$ (Marchi et al., 2016). Our findings relevant to $\mathrm{SEQ} \%_{\mathrm{CBR}}$ and $\mathrm{CBRS} \mathrm{SEQ}_{\mathrm{SE}}$ confirm well-known results present in literature and support the strong involvement of the $\mathrm{CBR}$ in governing the adjustment of cardiovascular variables. Conclusions held regardless the strategy followed to set $\tau_{\mathrm{HP}-\mathrm{SAP}}$ (i.e., $\tau_{\mathrm{HP}-\mathrm{SAP}}=0$ or the $\tau_{\mathrm{HP}-\mathrm{SAP}}$ optimization according to the maximum of the HP-SAP cross-correlation). Indeed, the gradual increase of $S E Q \%{ }_{C B R}$ with tilt table inclination is in agreement with the results reported in Bahjaoui-Bouhaddi et al. (2000), Porta et al. (2016) and with the rise of the strength of the HP-SAP association along the temporal direction from SAP to HP reported in Porta et al. (2011). The progressive decrease of $\mathrm{cBRS}_{\mathrm{SEQ}}$ with tilt table angles is in agreement with results given in Cooke et al. (1999), Bahjaoui-Bouhaddi et al. (2000), O'Leary et al. (2003), Porta et al. (2016) indicating that the more and more increased involvement of the cBR in governing HPSAP variability interactions with the magnitude of the postural challenge is accompanied by the progressive decline of the effectiveness of this reflex. The reduction of $\mathrm{cBRS}_{\mathrm{SEQ}}$ is likely to be related to the reduction of $\mathrm{HP}$ in presence of virtually unmodified SAP values and to the reduced HP variation resulting from the vagal withdrawal in presence of an increased SAP variability linked to the increased modulation of the sympathetic discharge.

\section{The Simultaneous Characterization of cBR and sBR}

$\mathrm{cBR}$ and $\mathrm{sBR}$ are arms of the same negative neural control feedback aiming at confining disproportionate variability of AP inside a more physiological range through the modulation of HP and sympathetic outflow, respectively (Sundlöf and Wallin, 1978; Hunt et al., 2001; Robertson et al., 2012). Although, the two arms share the same finalistic aim (i.e., controlling systemic AP), sensing areas (mainly carotid sinuses and aortic arch), sensed variable (i.e., systemic AP) and receptors (i.e., the high pressure baroreceptors responding to vascular wall stretch), it can be hypothesized that the two reflexes are not fully redundant (Taylor et al., 2015). Indeed, since the target variable, the efferent pathways and the levels of integration are diverse, $\mathrm{cBR}$, and $\mathrm{sBR}$ can take care of specific aspects of the baroreflex control. In agreement with these observations we found a significant relation between $s B R S_{S E Q}$ and $c B R S_{S E Q}$ but the detected degree of association cannot be considered strong (i.e., $r=-0.302, p=3.30 \times 10^{-2}$ ), thus indicating a certain degree of independence between sBR and cBR controls. This finding stresses the relevance of the contemporaneous monitoring of $\mathrm{sBR}$ and $\mathrm{cBR}$ to achieve a more complete and insightful characterization of the human baroreflex regulation. This observation is in agreement with (Taylor et al., 2015) who found a significant relation between cBRS and sBRS in healthy subjects at rest in supine condition using methods based on spontaneous variability (i.e., BTA and cBR sequence technique). The present study extends the results reported in Taylor et al. (2015) in situations of mild sympathetic activation such as those induced by head-up tilt with small tilt table inclinations. This conclusion is in disagreement with the results reported in Rudas et al. (1999) and Dutoit et al. (2010) reporting the uncorrelation between sBRS and cBRS. The origin of the disagreement might be related to the approach followed in Rudas et al. (1999) and Dutoit et al. (2010) who computed sBRS and cBRS according to pharmacological methods (i.e., the modified Oxford technique) producing results that are hardly comparable with those based on spontaneous fluctuations (Taylor et al., 2015). The presence of a significant correlation between $\mathrm{SEQ} \%_{\mathrm{cBR}}$ and $\mathrm{SEQ} \%_{\mathrm{sBR}}$, suggests that both $\mathrm{sBR}$ and $\mathrm{cBR}$ controls were simultaneously activated in proportion to the challenge. Remarkably, the number of detected $c B R$ and $s B R$ sequences was small compared to the total amount of sequences (i.e., SEQ\% ${ }_{c B R}$ and $S E Q \%_{s B R}$ were on average $<5$ and $9 \%$, respectively), thus indicating that $\mathrm{cBR}$ and $\mathrm{sBR}$, even though relevant cardiovascular regulations, operate with several other different mechanisms contributing to adjust HP and MSNA burst rate according to different logics.

\section{Limitations of the Study and Future Developments}

The procedure exploited to set the parameters for the detection of sBR sequences is inherently based on the hypothesis of a positive relation of $\mathrm{SEQ} \%_{\mathrm{sBR}}$ and $\mathrm{sBRS} \mathrm{SEQ}_{\mathrm{SE}}$ with tilt table inclination in keeping with, respectively, a more and more important involvement of sBR control and a reduction of the magnitude of its intervention with the relevance of the baroreflex unloading (Ichinose et al., 2006; Barbic et al., 2015; Marchi et al., 2015). While there is a broad agreement in the literature about the increased involvement of sBR control with the magnitude of the baroreflex unloading (O'Leary et al., 2003; Fu et al., 2006; Ichinose et al., 2006; Barbic et al., 2015), the reduction of sBRS with tilt table inclination is much more controversial. For example, there are some studies reporting more negative values of sBRS during head-up tilt compared to T0 (O'Leary et al., 2003; $\mathrm{Fu}$ et al., 2006). In our data sBRS was neither more negative 
during head-up tilt compared to T0 nor negatively related to tilt table angles. Conversely, our data supported the presence of a significant positive relation of sBRS with the magnitude of the orthostatic challenge. It is worth noting that in $\mathrm{Fu}$ et al. (2006) and O'Leary et al. (2003) nonpharmacological methods expressing sBRS in arbitrary units and requiring normalization were utilized. Future studies should test whether increasing further the relevance of the orthostatic stimulus by exposing the subject to tilt table inclinations of 75 and $90^{\circ}$ could reduce even further the sBRS. In addition, it remains to be established whether conclusions might depend on the selection of the sensed variable (e.g., the use of DAP in the study of sBR and of SAP in the study of $\mathrm{cBR}$ ). As a further limitation this study did not face the issue of the sparseness of MSNA and its possible effect on the sBR sequence method. We advocate a specific evaluation of this issue by explicitly comparing the characterization of $s B R$ in a group of subjects with sparse MSNA and a group with a higher tonic MSNA within the same experimental condition (e.g., during T0). Moreover, since this study is focused on methods of sBR characterization that do not require normalization because they are based on counting MSNA bursts and assessing its rate of occurrence such as the BTA and sBR sequence techniques, their eventual link with those requiring normalization procedures because they are based on MSNA low-pass filtering focusing the frequency content between 0.0 and $0.5 \mathrm{~Hz}$ (Saul et al., 1990; Pagani et al., 1997; Nakata et al., 1998; Taylor et al., 1998; Cooke et al., 1999; Furlan et al., 2000; Kamiya et al., 2005; Ryan et al., 2011) or on the evaluation of the amplitude or area of the MSNA bursts (Sundlöf and Wallin, 1978; Kienbaum et al., 2001) requires further studies. Another issue that deserves a specific

\section{REFERENCES}

Bahjaoui-Bouhaddi, M., Cappelle, S., Henriet, M.-T., Dumoulin, G., Wolf, J.-P., and Regnard, J. (2000). Graded vascular autonomic control versus discontinuous cardiac control during graded upright tilt. J. Auton. Nerv. Syst. 70, 140-155. doi: 10.1016/s0165-1838(99)00068-5

Barbic, F., Heusser, K., Marchi, A., Zamunér, A. R., Gauger, P., Tank, J., et al. (2015). Cardiovascular parameters and neural sympathetic discharge variability before orthostatic syncope: role of sympathetic baroreflex control to the vessels. Physiol. Meas. 36, 633-641. doi: 10.1088/0967-3334/36/4/633

Baselli, G., Cerutti, S., Badilini, F., Biancardi, L., Porta, A., Pagani, M., et al. (1994). Model for the assessment of heart period and arterial pressure variability interactions and respiratory influences. Med. Biol. Eng. Comput. 32, 143-152.

Baumert, M., Lambert, E., Vaddadi, G., Sari, C. I., Esler, M., Lambert, G., et al. (2011). Cardiac repolarization variability in patients with postural tachycardia syndrome during graded head-up tilt. Clin. Neurophysiol. 122, 405-409. doi: 10.1016/j.clinph.2010.06.017

Bertinieri, G., di Rienzo, M., Cavallazzi, A., Ferrari, A. U., Pedotti, A., and Mancia, G. (1985). A new approach to analysis of the arterial baroreflex. J. Hypertens. 3, S79-S81.

Cooke, W. H., Hoag, J. B., Crossman, A. A., Kuusela, T. A., Tahvanainen, K. U. O., and Eckberg, D. L. (1999). Human responses to upright tilt: a window on central autonomic integration. J. Physiol. 517, 617-628.

Diedrich, A., Porta, A., Barbic, F., Brychta, R. J., Bonizzi, P., Diedrich, L., et al. (2009). Lateralization of expression of neural sympathetic activity to the vessels and effects of carotid baroreceptor stimulation. Am. J. Physiol. 296, H1758H1765. doi: 10.1152/ajpheart.01045.2008

Dutoit, A. P., Hart, E. C., Charkoudian, N., Wallin, B. G., Curry, T. B., and Joyner, M. J. (2010). Cardiac baroreflex sensitivity is not correlated to study featuring a larger number of subjects and, thus, a higher statistical power, is the comparison between the characterization of $\mathrm{cBR}$ and $\mathrm{sBR}$ within a given experimental condition.

\section{Perspectives and Significance}

We proposed a causal method for the characterization of the sBR from spontaneous beat-to-beat variability of MSNA burst rate and DAP based on the definition and extraction of sequences of sBR origin. Since this proposed method follows the same logic as the $\mathrm{cBR}$ sequence technique, the contemporaneous exploitation of both techniques allows one to set a framework assuring a homogenous characterization of both $\mathrm{cBR}$ and $\mathrm{sBR}$, thus favoring studies aiming at understanding cardiovascular control and the degree of independence between the two baroreflex arms, e.g., in heart failure patients (Watson et al., 2007). In addition, the proposed method allows one to overcome some limitations of more traditional estimates of sBRS such as the administration of a pharmacological challenge in pharmacological approaches, the inherent need of long recordings in nonpharmacological applications, and the necessity of normalization procedures typical of methods based on computation of amplitude or area of the MSNA bursts.

\section{AUTHOR CONTRIBUTIONS}

AM analyzed the data; AM and AP drafted the manuscript; ME and EL performed the experiments; $\mathrm{AM}, \mathrm{VB}, \mathrm{BD}, \mathrm{ME}, \mathrm{EL}, \mathrm{MB}$, and $\mathrm{AP}$ interpreted the data; $\mathrm{AM}, \mathrm{VB}, \mathrm{BD}, \mathrm{ME}, \mathrm{EL}, \mathrm{MB}$, and $\mathrm{AP}$ revised the manuscript; $\mathrm{AM}, \mathrm{VB}, \mathrm{BD}, \mathrm{ME}, \mathrm{EL}, \mathrm{MB}$, and $\mathrm{AP}$ approved the final version of the manuscript. sympathetic baroreflex sensitivity within healthy young humans. Hypertension 56, 1118-1123. doi: 10.1161/HYPERTENSIONAHA.110.158329

Ebert, T. J., and Cowley, A. W. (1992). Baroreflex modulation of sympathetic outflow during physiological increases of vasopressin in humans. Am. J. Physiol. 262, H1372-H1378.

Eckberg, D. L. (1976). Temporal response patterns of the human sinus node to brief carotid baroreceptor stimuli. J. Physiol. 258, 769-782.

El-Hamad, F., Lambert, E., Abbott, D., and Baumert, M. (2015). Relation between QT interval variability and muscle sympathetic nerve activity in normal subjects. Am. J. Physiol. Heart Circ. Physiol. 309, H1218-H1224. doi: 10.1152/ajpheart.00230.2015

Fu, Q., Shook, R. P., Okazaki, K., Hastings, J. L., Shibata, S., Conner, C. L., et al. (2006). Vasomotor sympathetic neural control is maintained during sustained upright posture in humans. J. Physiol. 577, 679-687. doi: 10.1113/jphysiol.2006.118158

Furlan, R., Porta, A., Costa, F., Tank, J., Baker, L., Schiavi, R., et al. (2000). Oscillatory patterns in sympathetic neural discharge and cardiovascular variables during orthostatic stimulus. Circulation 101, 886-892. doi: 10.1161/01.CIR.101.8.886

Hamner, J. W., and Taylor, J. A. (2001). Automated quantification of sympathetic beat-to-beat activity, independent of signal quality. J. Appl. Physiol. 91, 1199-1206.

Hart, E. C., Joyner, M. J., Wallin, B. G., Karlsson, T., Curry, T. B., and Charkoudian, N. (2010). Baroreflex control of muscle sympathetic nerve activity: a nonpharmacological measure of baroreflex sensitivity. Am. J. Physiol. 298, H816-H822. doi: 10.1152/ajpheart.00924.2009

Hart, E. C., Wallin, G. B., Curry, T. B., Joyner, M. J., Karlsson, T., and Charkoudian, N. (2011). Hysteresis in the sympathetic baroreflex: role of baseline nerve activity. J. Physiol. 589(Pt 13), 3395-3404. doi: 10.1113/jphysiol.2011.208538 
Hunt, B. E., Fahy, L., Farquhar, W. B., and Taylor, J. A. (2001). Quantification of mechanical and neural components of vagal baroreflex in humans. Hypertension 37, 1362-1368. doi: 10.1161/01.HYP.37.6.1362

Ichinose, M., Saito, M., Fujii, N., Kondo, N., and Nishiyasu, T. (2006). Modulation of the control of muscle sympathetic nerve activity during severe orthostatic stress. J. Physiol. 576, 947-958. doi: 10.1113/jphysiol.2006.117507

Kamiya, A., Hayano, J., Kawada, T., Michikami, D., Yamamoto, K., Ariumi, H., et al. (2005). Low-frequency oscillation of sympathetic nerve activity decreases during development of tilt-induced syncope preceding sympathetic withdrawal and bradycardia. Am. J. Physiol. 289, H1758-H1769. doi: 10.1152/ajpheart.01027.2004

Keller, D. M., Cui, J., Davis, S. L., Low, D. A., and Crandall, C. G. (2006). Heat stress enhances arterial baroreflex control of muscle sympathetic nerve activity via increased sensitivity of burst gating, not burst area, in humans. J. Physiol. 573, 445-451. doi: 10.1113/jphysiol.2006.108662

Kienbaum, P., Karlsson, T., Sverrisdottir, Y. B., Elam, M., and Wallin, B. G. (2001). Two sites for modulation of human sympathetic activity by arterial baroreceptors. J. Physiol. 531, 861-869. doi: 10.1111/j.1469-7793.2001.0861h.x

Lambert, E., Eikelis, N., Esler, M., Dawood, T., Schlaich, M., Bayles, R., et al. (2008). Altered sympathetic nervous system reactivity and norepinephrine transporter expression in patients with postural tachycardia syndrome. Circ. Arrhythmia Electrophysiol. 1, 103-109. doi: 10.1161/CIRCEP.107.750471

Laude, D., Elghozi, J.-L., Girard, A., Bellard, E., Bouhaddi, M., Castiglioni, P., et al. (2004). Comparison of various techniques used to estimate spontaneous baroreflex sensitivity (the EuroBaVar study). Am. J. Physiol. 286, R226-R231. doi: 10.1152/ajpregu.00709.2002

Marchi, A., Bari, V., De Maria, B., Cerutti, S., Heusser, K., Tank, J., et al. (2015). Evaluation of the correlation between cardiac and sympathetic baroreflex sensitivity before orthostatic syncope. Conf. Proc. IEEE Eng. Med. Biol. Soc. 37, 2063-2066. doi: 10.1109/embc.2015.7318793

Marchi, A., Bari, V., De Maria, B., Esler, M., Lambert, E., Baumert, M., et al. (2016). Calibrated variability of muscle sympathetic nerve activity during graded headup tilt in humans and its link with noradrenaline data and cardiovascular rhythms. Am. J. Physiol. 310, R1134-R1143. doi: 10.1152/ajpregu. 00541.2015

Montano, N., Gnecchi-Ruscone, T., Porta, A., Lombardi, F., Pagani, M., and Malliani, A. (1994). Power spectrum analysis of heart rate variability to assess changes in sympatho-vagal balance during graded orthostatic tilt. Circulation 90, 1826-1831.

Nakata, A., Takata, S., Yuasa, T., Shimakura, A., Maruyama, M., Nagai, H., et al. (1998). Spectral analysis of heart rate, arterial pressure and muscle sympathetic nerve activity in normal humans. Am. J. Physiol. 274, H1211-H1217.

O'Leary, D. D., Kimmerly, D. S., Cechetto, A. D., and Shoemaker, J. K. (2003). Differential effect of head-up tilt on cardiovagal and sympathetic baroreflex sensitivity in humans. Exp. Physiol. 88, 769-774. doi: 10.1113/eph88 02632

Pagani, M., Montano, N., Porta, A., Malliani, A., Abboud, F. M., Birkett, C., et al. (1997). Relationship between spectral components of cardiovascular variabilities and direct measures of muscle sympathetic nerve activity in humans. Circulation 95, 1441-1448. doi: 10.1161/01.CIR.95.6.1441

Pickering, T. G., Gribbin, B., and Sleight, P. (1972). Comparison of the reflex heart rate response to rising and falling arterial pressure in man. Cardiovasc. Res. 6, 277-283.

Porta, A., Baselli, G., Rimoldi, O., Malliani, A., and Pagani, M. (2000). Assessing baroreflex gain from spontaneous variability in conscious dogs: role of causality and respiration. Am. J. Physiol. 279, H2558-H2567.

Porta, A., Catai, A. M., Takahashi, A. C. M., Magagnin, V., Bassani, T., Tobaldini, E., et al. (2011). Causal relationships between heart period and systolic arterial pressure during graded head-up tilt. Am. J. Physiol. 300, R378-R386. doi: 10.1152/ajpregu.00553.2010

Porta, A., Takahashi, A. C. M., and Catai, A. M. (2016). Cardiovascular coupling during graded postural challenge: comparison between linear tools and joint symbolic analysis. Braz. J. Phys. Therapy. doi: 10.1590/bjpt-rbf.2014.0179. [Epub ahead of print].

Porta, A., Tobaldini, E., Guzzetti, S., Furlan, R., Montano, N., and GnecchiRuscone, T. (2007). Assessment of cardiac autonomic modulation during graded head-up tilt by symbolic analysis of heart rate variability. Am. J. Physiol. 293, H702-H708. doi: 10.1152/ajpheart.00006.2007

Robertson, D., Diedrich, A., and Chapleau, M. W. (2012). Editorial on arterial baroreflex issue. Auton. Neurosci-Basic Clin. 172, 1-3. doi: 10.1016/j.autneu.2012.10.010

Rudas, L., Crossman, A. A., Morillo, C. A., Halliwill, J. R., Tahvanainen, K. U. O., Kuusela, T. A., et al. (1999). Human sympathetic and vagal baroreflex responses to sequential nitroprusside and phenylephrine. Am. J. Physiol. 276, H1691-H1698. doi: 10.1113/jphysiol.2011.213074

Ryan, K. L., Rickards, C. A., Hinojosa-Laborde, C., Cooke, W. H., and Convertino, V. A. (2011). Arterial pressure oscillations are not associated with muscle sympathetic nerve activity in individuals exposed to central hypovolaemia. $J$. Physiol. 589, 5311-5322. doi: 10.1113/jphysiol.2011.213074

Saul, J. P., Rea, R. F., Eckberg, D. L., Berger, R. D., and Cohen, R. J. (1990). Heart rate muscle sympathetic nerve variability during reflex changes of autonomic activity. Am. J. Physiol. 258, H713-H721.

Smyth, H. S., Sleight, P., and Pickering, G. W. (1969). Reflex regulation of the arterial pressure during sleep in man. A quantitative method of assessing baroreflex sensitivity. Circ. Res. 24, 109-121.

Studinger, P., Goldstein, R., and Taylor, J. A. (2009). Age- and fitness-related alterations in vascular sympathetic control. J. Physiol. 587, 2049-2057. doi: 10.1113/jphysiol.2009.170134

Sundlöf, G., and Wallin, B. G. (1978). Human muscle nerve sympathetic activity at rest. Relationship to blood pressure and age. J. Physiol. 274, 621-637.

Taylor, C. E., Witter, T., El Sayed, K., Hissen, S. L., Johnson, A. W., and Macefield, V. G. (2015). Relationship between spontaneous sympathetic baroreflex sensitivity and cardiac baroreflex sensitivity in healthy young individuals. Physiol. Rep. 3:e12536. doi: 10.14814/phy2.12536

Taylor, J. A., Williams, T. D., Seals, D. R., and Davy, K. P. (1998). Low-frequency arterial pressure fluctuations do not reflect sympathetic outflow: gender and age differences. Am. J. Physiol. 274, H1194-H1201.

Wallin, B. G., Burke, D., and Gandevia, S. (1994). Coupling between variations in strength and baroreflex latency of sympathetic discharges in human muscle nerves. J. Physiol. 474, 331-338.

Watson, A. M. D., Hood, S. G., Ramchandra, R., McAllen, R. M., and May, C. N. (2007). Increased cardiac sympathetic nerve activity in heart failure is not due to desensitization of the arterial baroreflex. Am. J. Physiol. 293, H798-H804. doi: 10.1152/ajpheart.00147.2007

Conflict of Interest Statement: The authors declare that the research was conducted in the absence of any commercial or financial relationships that could be construed as a potential conflict of interest.

Copyright (c) 2016 Marchi, Bari, De Maria, Esler, Lambert, Baumert and Porta. This is an open-access article distributed under the terms of the Creative Commons Attribution License (CC BY). The use, distribution or reproduction in other forums is permitted, provided the original author(s) or licensor are credited and that the original publication in this journal is cited, in accordance with accepted academic practice. No use, distribution or reproduction is permitted which does not comply with these terms. 